\title{
CONLEY DECOMPOSITION FOR CLOSED RELATIONS
}

\author{
R. P. MCGEHEE \& T. WIANDT
}

\begin{abstract}
This paper presents a theory of dynamics of closed relations on compact Hausdorff spaces. It contains an investigation of set valued maps and establishes generalizations for some topological aspects of dynamical systems theory, including recurrence, attractor-repeller structure and the Conley Decomposition Theorem.
\end{abstract}

\section{INTRODUCTION}

The Conley Decomposition Theorem [3] is one of the most fundamental theorems in the theory of dynamical systems. In the original setting of flows on compact metric spaces, Conley introduced a very weak form of recurrence, which he called "chain recurrence". Roughly speaking, a point is chain recurrent if it returns to itself by following the flow for an arbitrarily long time, making arbitrarily small jumps, or errors, along the way. He then proved the existence of what he termed a "Liapunov function", a real-valued function strictly decreasing everywhere except on components of the chain-recurrent set, where it is constant.

Our main point is to argue that the natural setting for the Conley Decomposition Theorem is that of iterations of closed relations on compact spaces. (I.e. iterations of closed subsets of $X \times X$ in the product topology.) This case has already been made by Akin [1]. We provide here more evidence.

The reader may ask what relations have to do with dynamical systems. The theory of dynamical systems is the study of how systems evolve with time. In the early history of the subject, time was taken to be continuous, and the evolution of the system was described by differential equations. The theory was quickly extended to include discrete time systems, where the evolution of the system was modeled by iteration of a map $f$, invertible at first, later noninvertible. If the state of the system at time $t$ is given by $x$, then the state of the system at time $t+1$ is given by $f(x)$ and the state of the system at time $t+k$ is given by $f^{k}(x)$. If the map $f$ is invertible, then one can follow the system backward in time via the inverse of the map, yielding $f^{-k}(x)$ as the state of the system at time $t-k$.

If the map $f$ is noninvertible, then following the system backward in time requires some interpretation. A point can have a unique preimage, as is the case for an invertible map, or it can have many preimages, or it can have no preimage. Following the system backward in time may be possible in a unique way, it may be possible in many ways, or it may be impossible. However, most of the concepts used in the study of invertible maps have reasonable extensions. For example, an "orbit" for an invertible map is a bi-infinite sequence $\left(\ldots, f^{-1}(x), x, f(x), f^{2}(x), \ldots\right)$. For a noninvertible map, an orbit becomes a sequence $\left(\ldots, x_{-1}, x_{0}, x_{1}, x_{2}, \ldots\right)$, either finite or infinite, such that $x_{k+1}=f\left(x_{k}\right)$. If the sequence is finite, it is possible to

Date: 16 March 2005. 
extend it forward in time to a semi-infinite sequence, but it may not be possible to extend it backward.

This definition of orbit works just as well for multivalued maps or relations. A relation on a space $X$ is simply a subset $f$ of $X \times X$. Note that the graph of a function is a special case of a relation. For a relation, we write $y=f(x)$ as equivalent to $(x, y) \in f$. An orbit is once again a sequence $\left(\ldots, x_{-1}, x_{0}, x_{1}, x_{2}, \ldots\right)$, either finite or infinite, such that $x_{k+1}=f\left(x_{k}\right)$. In this case, however, it may not be possible to extend the sequence either forward or backward in time.

Relations can be composed and hence iterated. If $f$ and $g$ are both relations on $X$, then the composition of $f$ and $g$ is the relation

$$
f \circ g=\{(x, z) \in X \times X:(x, y) \in g \text { and }(y, z) \in f \text { for some } y \in X\} .
$$

We see that $(x, y) \in f^{n}$ if and only if there is an orbit of length $n$ from $x$ to $y$.

As we discuss below in detail, following a relation backward in time is equivalent to iterating the transpose of the relation. The transpose of a relation $f$ is the set $\{(y, x):(x, y) \in f\}$, which is itself another relation. Note that the transpose of the graph of an invertible map is the graph of the inverse of the map.

This discussion of orbits and iteration of relations illustrates one of the main points of this paper: the extension of the theory of dynamical systems from invertible to noninvertible maps introduces a mathematically unnatural asymmetry that is corrected by further extending the theory to relations. A noninvertible map does not have an inverse, and following the system backward in time is very different from following it forward in time. However, the graph of a noninvertible map always has a transpose, and following the system backward in time is equivalent to iterating this transpose as a relation. So we may as well start with relations in the first place. Since the transpose of a relation is again a relation, the symmetry between forward and backward iteration is restored.

One very well studied example for one-dimensional dynamics is the family of quadratic maps $f_{\mu}(x)=\mu x(1-x)$ on $[0,1]$. This noninvertible map can be viewed as a relation on $[0,1] \times[0,1]$,

$$
f_{\mu}=\{(x, y): y=\mu x(1-x)\}
$$

This is a closed relation in the product topology. Its transpose is also a closed relation,

$$
f_{\mu}^{*}=\{(x, y): x=\mu y(1-y)\}
$$

We will investigate this example in more detail in Section 15.

The concept of a "pseudo-orbit" also fits naturally into the setting of relations. An $\varepsilon$-pseudo-orbit for a map $f$ on a metric space $X$ is a finite or infinite sequence satisfying $d\left(f\left(x_{k}\right), x_{k+1}\right) \leq \varepsilon$. For maps, a point is called "chain-recurrent" if there is an $\varepsilon$-pseudo-orbit from the point back to itself for arbitrarily small positive $\varepsilon$. One can fatten up the graph of $f$ to a relation $g$ by letting

$$
g=\{(x, y): d(y, f(x)) \leq \varepsilon\} .
$$

Note that an orbit for the relation $g$ is exactly an $\varepsilon$-pseudo-orbit for the map $f$. Once again, the existing theory of dynamical systems leads naturally to the study of relations.

Conley himself introduced a relation into his development of the decomposition theorem. A point $y$ is related to a point $x$ if there exists an $\varepsilon$-pseudo-orbit from $x$ 
to $y$ for arbitrarily small positive $\varepsilon$. Thus a point is chain-recurrent if and only if it is related to itself by the Conley relation.

For a map $f$ on a space $X$, the associated Conley relation is indeed a relation on $X$. As we show below, the notion of the Conley relation can be extended to the case when $f$ is itself a relation on $X$, yielding again a relation on $X$. (See Section 8.) Starting with relations instead of maps leads to a satisfying mathematical completeness.

We like to think of the Conley relation as the "infinite iterate" of the map or relation, since it provides information about the ultimate behavior of the dynamical system as time goes to infinity. This thought is made more precise below, where we denote the Conley relation for $f$ by $f^{\Omega}$. In the process of developing this notation, we introduce two more relations $f^{\infty}$ and $f^{\omega}$, which can also be thought of as "infinite iterates". Roughly speaking, all three relations $f^{\infty}, f^{\omega}$, and $f^{\Omega}$ provide information about the ultimate behavior of the iterates of $f$. The relation $f^{\infty}$ takes no topology into account, whereas $f^{\omega}$ takes into account the topology of the state space, and $f^{\Omega}$ takes into account a topology on the space of relations.

The relations $f^{\infty}$ and $f^{\omega}$ are interesting in their own right. As mentioned above, a point is chain recurrent if and only if it is related to itself by the Conley relation. In other words, the chain recurrent set is exactly the fixed point set of the relation $f^{\Omega}$. We show below that the fixed point set of $f^{\infty}$ is the set of periodic points, while the fixed point set of $f^{\omega}$ is the nonwandering set.

Relations have been used by other authors in the study of dynamical systems. Akin [1] developed a general theory for iterations of relations on compact metric spaces. A theory of entropy for relations was created by Langevin, Walczak and Przyticky [6, 7]. Barnsley [2] studied fractals generated by contraction mapping systems, which are a special class of relations. McGehee and Sander [9] gave a new proof of the stable manifold theorem using the abstract setting of relations. Sander [11] developed a notion for hyperbolicity for noninvertible maps and relations.

The work for this paper began while McGehee was visiting the University of Colorado in 1990. McGehee gratefully acknowledges the support of the Ulam Professorship program at Colorado. The work evolved over the years, and both McGehee and Wiandt received support from the Geometry Center at the University of Minnesota.

Although not presented in this paper, Wiandt has established the existence of a Liapunov function for any closed relation on a second countable compact Hausdorff space [13], thus completing the extension of Conley's theorem to this very general setting.

In this paper, first we develop basic notions about set-valued maps. We will need these results later, because relations generate set-valued maps in a natural way. After obtaining these basic results, we will investigate what properties these relation-generated set-valued maps have and we also generalize some of the basic concepts of dynamical systems theory for the setting of relations. In Sections 6-8 the fundamental constructions are introduced. These constructions are the main tools in the investigation of limit behavior and recurrence. In Sections 10-13 we exploit the previous constructions again to develop the theory about attractors and repellers and we prove the generalization of Conley's decomposition theorem. The last two sections contain some simple illustrating examples. 


\section{Set-VAlued MAPS}

In this section we formulate the setting of the paper and give the motivation for the study of set-valued maps, then we state some elementary results as well.

Definition 2.1. A relation on a set $X$ is a subset of $X \times X$. The set of all relations on $X$ will be denoted by $\mathcal{R}(X)$.

Definition 2.2. If $f$ is a relation on $X$ and $S \subset X$, then the image of $S$ under $f$ is the set

$$
f(S) \equiv\{y \in X: \text { there exists } x \in S \text { satisfying }(x, y) \in f\} .
$$

A relation $f$ on a set $X$ therefore can be thought of as a set-valued function on $X$. A slightly different viewpoint is that a relation $f$ on a set $X$ induces a map on the set of subsets of $X$, denoted by $2^{X}$ throughout the paper. (Note that $\mathcal{R}(X)=2^{X \times X}$. We will use both notations in the paper.)

Definition 2.3. If $f$ is a relation on $X$, then the induced map $f$ is the map

$$
f: 2^{X} \rightarrow 2^{X}: S \mapsto f(S) .
$$

In order to examine the behavior of relations on sets, the previous definition suggests that first we develop some theory about maps on the set of subsets of a set. We will define now some elementary concepts and basic notions. First, we will deal only with general sets without a topological structure, then we establish important notions in the case when $X$ is a topological space.

The first lemma is an elementary result about intersections.

Lemma 2.4. If $\mathfrak{S} \subset 2^{X}$ and $\mathfrak{T} \subset 2^{X}$, then the following statements hold.

(a) If $\mathfrak{S} \subset \mathfrak{T}$, then $\bigcap \mathfrak{T} \subset \bigcap \mathfrak{S}$.

(b) If for every $T \in \mathfrak{T}$ there exists an $S \in \mathfrak{S}$ such that $S \subset T$, then $\bigcap \mathfrak{S} \subset \bigcap \mathfrak{T}$.

We will now define some self-explanatory notions for general maps on the set of subsets of a set. Although relations induce maps from $2^{X}$ to $2^{X}$, we will state our results a little bit more generally, i.e. we will consider maps from $2^{X}$ to $2^{Y}$, where $X$ and $Y$ are (possibly) different sets. This way we will be able to apply our results later at the construction of the composition map.

Definition 2.5. A map $\Theta: 2^{X} \rightarrow 2^{Y}$ is said to preserve inclusion if and only if $\Theta(S) \subset \Theta(T)$ whenever $S \subset T \subset X$. The map is said to preserve union if and only if for all $\mathfrak{S} \subset 2^{X}$

$$
\Theta(\bigcup \mathfrak{S})=\bigcup\{\Theta(S): S \in \mathfrak{S}\}
$$

The map is said to preserve intersection if and only if for all $\mathfrak{S} \subset 2^{X}$

$$
\Theta(\bigcap \mathfrak{S})=\bigcap\{\Theta(S): S \in \mathfrak{S}\} \text {. }
$$

The following lemma is an immediate consequence of the definitions.

Lemma 2.6. The map $\Theta: 2^{X} \rightarrow 2^{Y}$ preserves inclusion if and only if for all $\mathfrak{S} \subset 2^{X}$

$$
\Theta(\bigcap \mathfrak{S}) \subset \bigcap\{\Theta(S): S \in \mathfrak{S}\}
$$

Now if we have a map $f: X \rightarrow Y$, then this map induces the following wellknown map on the set of subsets:

$$
f^{-1}: 2^{Y} \rightarrow 2^{X}: S \mapsto\{x: f(x) \in S\}
$$


Also, it will not cause any confusion if we denote another induced set-valued map, namely

$$
f: 2^{X} \rightarrow 2^{Y}: S \mapsto f(S)
$$

with the same letter $f$. We will need the following simple fact later.

Lemma 2.7. If $f: X \rightarrow Y$ is a map, $S \subset X$ and $T \subset Y$, then

$$
f\left(f^{-1}(T)\right) \subset T \text { and } S \subset f^{-1}(f(S)) .
$$

The next elementary lemma can be deduced easily from the definitions.

Lemma 2.8. If $f: X \rightarrow Y$ is a map, then $f^{-1}$ preserves inclusion, union and intersection.

The usual "dot" notation will be used for two-variable maps: fixing one variable, the dot denotes the place of the variable in the resulting one-variable map.

Definition 2.9. A map $\Theta: 2^{X} \times 2^{X} \rightarrow 2^{Y}$ is said to preserve inclusion if and only if for all $S \subset X$ the maps $\Theta(\cdot, S)$ and $\Theta(S, \cdot)$ preserve inclusion. The map is said to preserve union if and only if for all $S \subset X$ the maps $\Theta(\cdot, S)$ and $\Theta(S, \cdot)$ preserve union. The map is said to preserve intersection if and only if for all $S \subset X$ the maps $\Theta(\cdot, S)$ and $\Theta(S, \cdot)$ preserve intersection.

The next four lemmas follow readily from the definitions.

Lemma 2.10. The map $\Theta: 2^{X} \times 2^{X} \rightarrow 2^{Y}$ preserves inclusion if and only if $\Theta\left(S_{1}, S_{2}\right) \subset \Theta\left(T_{1}, T_{2}\right)$ whenever $S_{1} \subset T_{1} \subset X$ and $S_{2} \subset T_{2} \subset X$.

Lemma 2.11. The map $\Theta: 2^{X} \times 2^{X} \rightarrow 2^{Y}$ preserves inclusion if and only if for all $\mathfrak{S} \subset 2^{X}$ and $\mathfrak{T} \subset 2^{X}$

$$
\Theta(\bigcap \mathfrak{S}, \bigcap \mathfrak{T}) \subset \bigcap\{\Theta(S, T): S \in \mathfrak{S} \text { and } T \in \mathfrak{T}\} .
$$

Lemma 2.12. The map $\Theta: 2^{X} \times 2^{X} \rightarrow 2^{Y}$ preserves union if and only if for all $\mathfrak{S} \subset 2^{X}$ and $\mathfrak{T} \subset 2^{X}$

$$
\Theta(\bigcup \mathfrak{S}, \bigcup \mathfrak{T})=\bigcup\{\Theta(S, T): S \in \mathfrak{S} \text { and } T \in \mathfrak{T}\} .
$$

Lemma 2.13. The map $\Theta: 2^{X} \times 2^{X} \rightarrow 2^{Y}$ preserves intersection if and only if for all $\mathfrak{S} \subset 2^{X}$ and $\mathfrak{T} \subset 2^{X}$

$$
\Theta(\bigcap \mathfrak{S}, \bigcap \mathfrak{T})=\bigcap\{\Theta(S, T): S \in \mathfrak{S} \text { and } T \in \mathfrak{T}\} .
$$

From now on, we will assume that $X$ and $Y$ are topological spaces. The additional structure will allow us to introduce new important notions for maps on the set of subsets.

First we introduce the usual topological notations and define the neighborhoodstructure.

If $S$ is a subset of a topological space $X$, then the closure of $S$ is denoted by $\bar{S}$, the interior of $S$ is denoted by $S^{o}$ and the complement of $S$ is denoted by $S^{c}$. A neighborhood of $S$ is a set $U$ containing $S$ in its interior. That is, there exists an open set $V$, such that $S \subset V \subset U$. If $U$ is itself open, it is called an open neighborhood of $S$; if $U$ is closed, it is called a closed neighborhood of $S$.

For a given subset $S$ of the topological space $X$ the set of open neighborhoods will be denoted by $\mathfrak{N}^{\circ}(S)$, the set of neighborhoods by $\mathfrak{N}(S)$ and the set of closed neighborhoods by $\overline{\mathfrak{N}}(S)$.

Before stating the results, we need one more construction. 
Definition 2.14. If $\mathfrak{S}$ is a family of subsets of a fixed set, then $\mathfrak{S}$ is called directed if and only if the following statement holds. If $S_{1} \in \mathfrak{S}$ and $S_{2} \in \mathfrak{S}$, then there exists $S_{3} \in \mathfrak{S}$ satisfying $S_{3} \subset S_{1} \cap S_{2}$.

Now we can start to establish the basics of set valued maps on topological spaces.

Definition 2.15. If $X$ and $Y$ are topological spaces, then a map $\Theta: 2^{X} \rightarrow 2^{Y}$ is said to preserve open sets if and only if $\Theta(S)$ is open whenever $S \subset X$ is open. The map is said to preserve closed sets if and only if $\Theta(S)$ is closed whenever $S \subset X$ is closed.

The next notion is fundamental.

Definition 2.16. If $X$ and $Y$ are topological spaces, then a map $\Theta: 2^{X} \rightarrow 2^{Y}$ is called semicontinuous if and only if it satisfies the following conditions.

(a) $\Theta$ preserves inclusion.

(b) $\Theta$ preserves closed sets.

(c) If $\mathfrak{K}$ is a directed family of closed subsets of $X$, then

$$
\Theta(\bigcap \mathfrak{K})=\bigcap\{\Theta(K): K \in \mathfrak{K}\} .
$$

The following lemma regards the composition of semicontinuous maps.

Lemma 2.17. If $X, Y$ and $Z$ are topological spaces and $\Theta: 2^{X} \rightarrow 2^{Y}$ and $\Psi: 2^{Y} \rightarrow 2^{Z}$ are semicontinuous, then so is $\Psi \circ \Theta$.

Proof. Properties (a) and (b) are immediate, to check property (c) we only have to prove that if $\mathfrak{K}$ is a directed family of closed subsets, then $\{\Theta(K): K \in \mathfrak{K}\}$ is also a directed family of closed subsets. But this is true because of properties (a) and (b) for $\Theta$.

We will also need the two-variable version of these properties.

Definition 2.18. If $X$ and $Y$ are topological spaces, then a map $\Theta: 2^{X} \times 2^{X} \rightarrow 2^{Y}$ is said to preserve open sets if and only if for all open $S \subset X$ the maps $\Theta(\cdot, S)$ and $\Theta(S, \cdot)$ preserve open sets. The map is said to preserve closed sets if and only if for all closed $S \subset X$ the maps $\Theta(\cdot, S)$ and $\Theta(S, \cdot)$ preserve closed sets.

Definition 2.19. If $X$ and $Y$ are topological spaces, then a map $\Theta: 2^{X} \times 2^{X} \rightarrow 2^{Y}$ is called semicontinuous if and only if it satisfies the following conditions.

(a) $\Theta$ preserves inclusion.

(b) $\Theta$ preserves closed sets.

(c) If $\mathfrak{K}$ and $\mathfrak{L}$ are directed families of closed subsets of $X$, then

$$
\Theta(\bigcap \mathfrak{K}, \bigcap \mathfrak{L})=\bigcap\{\Theta(K, L): K \in \mathfrak{K} \text { and } L \in \mathfrak{L}\} .
$$

The next lemma is the two-variable counterpart of Lemma 2.17.

Lemma 2.20. If $X, Y$ and $Z$ are topological spaces and $\Theta: 2^{X} \times 2^{X} \rightarrow 2^{Y}$ and $\Psi: 2^{Y} \rightarrow 2^{Z}$ are semicontinuous, then so is $\Psi \circ \Theta$.

Proof. It is easy to check that $\Psi \circ \Theta$ preserves inclusion and closed sets. Let $\mathfrak{K}$ and $\mathfrak{L}$ be directed families of closed subsets of $X$. By properties (a) and (b) for $\Theta$ and Lemma $2.4\{\bigcap\{\Theta(K, L): L \in \mathfrak{L}\}: K \in \mathfrak{K}\}$ is a directed family of closed subsets. Because $\Theta$ preserves inclusion, we also know $\{\Theta(K, L): L \in \mathfrak{L}\}$ is a directed family for any $K \subset X$. These imply that

$$
\Psi(\Theta(\bigcap \mathfrak{K}, \bigcap \mathfrak{L}))=\bigcap\{\Psi(\Theta(K, L)): K \in \mathfrak{K} \text { and } L \in \mathfrak{L}\}
$$


and we verified property (c) for $\Psi \circ \Theta$.

The next two lemmas follow immediately from the definitions and Lemma 2.13.

Lemma 2.21. If $X$ and $Y$ are topological spaces and $\Theta: 2^{X} \rightarrow 2^{Y}$ preserves inclusion, intersection and closed sets, then $\Theta$ is semicontinuous.

Lemma 2.22. If $X$ and $Y$ are topological spaces and $\Theta: 2^{X} \times 2^{X} \rightarrow 2^{Y}$ preserves inclusion, intersection and closed sets, then $\Theta$ is semicontinuous.

The convenient choice of topology for our investigation will be compact Hausdorff topologies. We recall that these spaces are normal and compact subsets are closed and vice versa. Also, the (closed) neighborhoods of a fixed set form a directed family of (closed) subsets.

The proof of the following two lemmas are elementary.

Lemma 2.23. If $\mathfrak{S}$ is a directed family of closed subsets of a compact Hausdorff space $X$ and $K \subset \bigcap \mathfrak{S}$, then the following statements are equivalent.

(a) $K=\bigcap \mathfrak{s}$.

(b) If $V \in \mathfrak{N}(K)$, then there exists $S \in \mathfrak{S}$ such that $V \in \mathfrak{N}(S)$.

Lemma 2.24. If $K$ is a closed subset of a compact Hausdorff space $X$, then

$$
K=\bigcap \overline{\mathfrak{N}}(K) .
$$

The next theorem sheds light on the name "semicontinuous" and gives a characterization for this property.

Theorem 2.25. If $X$ and $Y$ are compact Hausdorff spaces and $\Theta: 2^{X} \rightarrow 2^{Y}$ preserves inclusion and closed sets, then the following two statements are equivalent.

(a) $\Theta$ is semicontinuous.

(b) If $W \in \overline{\mathfrak{N}}(\Theta(S))$, where $S \subset X$ is closed, then there exists $U \in \overline{\mathfrak{N}}(S)$ such that $\Theta(U) \subset W$.

Proof. (a) $\Rightarrow$ (b): Assume $\Theta$ is semicontinuous, let $S \subset X$ be a closed set and $W \subset Y$ satisfy $W \in \overline{\mathfrak{N}}(\Theta(S))$. Now since $\overline{\mathfrak{N}}(S)$ is a directed family satisfying $\bigcap \overline{\mathfrak{N}}(S)=S$ by Lemma 2.24 and since $\Theta$ is semicontinuous, it follows that

$$
\Theta(S)=\bigcap_{U \in \overline{\mathfrak{N}}(S)} \Theta(U)
$$

Since $\Theta$ preserves inclusion and closed sets, the set $\{\Theta(U): U \in \overline{\mathfrak{N}}(S)\}$ is also a directed family of closed sets, but then Lemma 2.23 implies the existence of $U \in \overline{\mathfrak{N}}(S)$ such that $\Theta(U) \subset W$, and the implication (a) $\Rightarrow(\mathrm{b})$ is established.

(b) $\Rightarrow$ (a): Let $\mathfrak{K}$ be a directed family of closed subsets of $X$, and let $K \equiv \bigcap \mathfrak{K}$. Since $\Theta$ preserves inclusion, the inclusion

$$
\Theta(K) \subset \bigcap_{L \in \mathfrak{K}} \Theta(L)
$$

follows immediately. Since $\Theta$ preserves closed sets, $\Theta(K)$ is closed and Lemma 2.24 implies that

$$
\Theta(K)=\bigcap_{W \in \overline{\mathfrak{N}}(\Theta(K))} W .
$$


Now if $W \in \overline{\mathfrak{N}}(\Theta(K))$, then by assumption there exists a $U \in \overline{\mathfrak{N}}(K)$ such that $\Theta(U) \subset W$. Since $U \in \overline{\mathfrak{N}}(K)$ and $K=\bigcap \mathfrak{K}$, by Lemma 2.23 there exists $L \in \mathfrak{K}$, such that $U \in \overline{\mathfrak{N}}(L)$. Then $\Theta(L) \subset \Theta(U) \subset W$ and by Lemma 2.4 we get

$$
\bigcap_{L \in \mathfrak{K}} \Theta(L) \subset \bigcap_{W \in \overline{\mathfrak{N}}(\Theta(K))} W=\Theta(K),
$$

which, with inclusion (1), implies that

$$
\bigcap_{L \in \mathfrak{K}} \Theta(L)=\Theta(K)=\Theta(\bigcap \mathfrak{K}),
$$

i.e., $\Theta$ is semicontinuous. The implication (b) $\Rightarrow(a)$ is established, and the proof is complete.

We close this section with the following theorem, which will be very important later.

Theorem 2.26. If $X$ and $Y$ are compact Hausdorff spaces and $f: X \rightarrow Y$ is a continuous map, then the induced map $f: 2^{X} \rightarrow 2^{Y}: S \mapsto f(S)$ is semicontinuous.

Proof. The induced map preserves inclusion and since $X$ is compact, $f$ is continuous and $Y$ is Hausdorff, the induced map preserves closed sets as well. Now let $S \subset X$ be closed and $W \subset Y$ satisfy $W \in \overline{\mathfrak{N}}(f(S))$. By definition, $f(S) \subset W^{o}$. Since $f$ is continuous, $U \equiv f^{-1}\left(W^{o}\right)$ is open. Now by Lemmas 2.7 and 2.8

$$
S \subset f^{-1}(f(S)) \subset f^{-1}\left(W^{o}\right)=U,
$$

which implies $S \cap U^{c}=\emptyset$. This means $S$ and $U^{c}$ are disjoint closed sets in a normal space, so there exist open sets $V$ and $T$ in $X$, such that $S \subset T, U^{c} \subset V$ and $V \cap T=\emptyset$. Then $V^{c}$ is closed and

$$
S \subset T \subset V^{c} \subset U,
$$

where $T$ is open, but this means $V^{c} \in \overline{\mathfrak{N}}(S)$ and using Lemmas 2.7 and 2.8 again we conclude

$$
f\left(V^{c}\right) \subset f(U)=f\left(f^{-1}\left(W^{o}\right)\right) \subset W^{o} \subset W .
$$

Therefore, the induced map $f$ satisfies statement (b) of Theorem 2.25, which implies that $f$ is semicontinuous and completes the proof.

\section{Relations ON SETS}

In this section we recall the setting of the paper and state some elementary results for relations on arbitrary sets. These results also can be found in [8]. We saw the definition of a relation on a set $X$ and the definition of the image of a subset of $X$ under a relation. The image of a set consisting of a single point occurs often enough to warrant the following specific notation: $f(x) \equiv f(\{x\})$.

We also saw how the relation induces a map on the set of subsets. It is easy to check that this induced map preserves inclusion and union.

Lemma 3.1. If $f$ is a relation on $X$, then the induced map $f$ preserves inclusion and union. 
The standard projection maps will be useful in the discussion. For $i=1,2$ denote

$$
\pi_{i}: X \times X \rightarrow X:\left(x_{1}, x_{2}\right) \mapsto x_{i}
$$

The following lemmas are elementary, they can be checked easily by the definitions.

Lemma 3.2. If $f$ is a relation on $X$ and $S \subset X$, then

$$
f(S)=\pi_{2}\left(\pi_{1}^{-1}(S) \cap f\right) .
$$

Lemma 3.3. If $f$ is a relation on $X, S \subset X$ and $T \subset X$, then $f(S) \subset T$ if and only if $\left(S \times T^{c}\right) \cap f=\emptyset$.

Lemma 3.4. If $f$ and $g$ are relations on $X$ and $S \subset X$, then $g(S) \subset f(S)$ whenever $g \subset f$.

Lemma 3.5. If $\mathcal{F} \subset \mathcal{R}(X)$ and $S \subset X$, then the following properties hold.

(a) $(\bigcup \mathcal{F})(S)=\bigcup\{f(S): f \in \mathcal{F}\}$.

(b) $(\bigcap \mathcal{F})(S)=\bigcap\{f(S): f \in \mathcal{F}\}$.

The following construction will be used extensively throughout the paper.

Definition 3.6. If $f$ is a relation on $X$ and $S \subset X$, then the inverse image of $S$ is the set

$$
f^{-1}(S) \equiv\{x \in X: f(x) \subset S\}
$$

Remark 3.7. In general, $f^{-1}$ is not generated by a relation. We can check this by considering the following example. Let $X \equiv\{0,1\}, f \equiv\{(0,0),(0,1),(1,0)\}$, $S_{0} \equiv\{0\}$, and $S_{1} \equiv\{1\}$. Were $f^{-1}$ generated by a relation, Lemma 3.1 would imply that $f^{-1}\left(S_{0} \cup S_{1}\right)=f^{-1}\left(S_{0}\right) \cup f^{-1}\left(S_{1}\right)$. However, $f^{-1}\left(S_{0} \cup S_{1}\right)=f^{-1}(Y)=X$, while $f^{-1}\left(S_{0}\right) \cup f^{-1}\left(S_{1}\right)=\{1\} \cup \emptyset=\{1\} \neq X$.

We will need the following elementary result later. Notice this is a version of Lemma 2.7 for relations.

Lemma 3.8. If $f$ is a relation on $X$ and $S \subset X$, then

$$
f\left(f^{-1}(S)\right) \subset S \subset f^{-1}(f(S)) .
$$

The next step is to find an "inverse" to the relation in the set of relations. This will be the transpose.

Definition 3.9. If $f$ is a relation on $X$, then the transpose of $f$ is the relation $f^{*}$ on $X$ defined by

$$
f^{*} \equiv\{(y, x) \in X \times X:(x, y) \in f\} .
$$

The following two lemmas are elementary.

Lemma 3.10. If $f$ and $g$ are relations on $X$, then the following properties hold.

(a) $\left(f^{*}\right)^{*}=f$.

(b) If $g \subset f$, then $g^{*} \subset f^{*}$.

Lemma 3.11. If $\mathcal{F} \subset \mathcal{R}(X)$, then the following properties hold.

(a) $(\bigcup \mathcal{F})^{*}=\bigcup\left\{f^{*}: f \in \mathcal{F}\right\}$.

(b) $(\bigcap \mathcal{F})^{*}=\bigcap\left\{f^{*}: f \in \mathcal{F}\right\}$. 
The inverse image of a set and the image of the set under the transpose are not identical in general, but they are related by the equality given by the following lemma.

Lemma 3.12. If $f$ is a relation on $X$ and $S \subset X$, then

$$
f^{-1}(S)^{c}=f^{*}\left(S^{c}\right)
$$

Later on, we will iterate relations. To do this, we must be able to compose them. The following definition is the customary generalization of the definition of composition of maps.

Definition 3.13. If $f$ and $g$ are relations on $X$, then the composition of $g$ with $f$ is the relation $g \circ f$ on $X$ defined by

$$
g \circ f \equiv\{(x, z) \in X \times X: \exists y \in X \text { such that }(x, y) \in f \text { and }(y, z) \in g\} .
$$

The next two lemmas will be important for the iteration of relations.

Lemma 3.14. If $f, g$ and $h$ are relations on $X$, then

$$
(h \circ g) \circ f=h \circ(g \circ f) .
$$

Lemma 3.15. If $f$ and $g$ are relations on $X$ and $S \subset X$, then

$$
(g \circ f)(S)=g(f(S))
$$

We have the following elementary result for the transpose of compositions.

Lemma 3.16. If $f$ and $g$ are relations on $X$, then

$$
(g \circ f)^{*}=f^{*} \circ g^{*} .
$$

Definition 3.17. The map

$$
\mathcal{R}(X) \times \mathcal{R}(X) \rightarrow \mathcal{R}(X):(f, g) \mapsto g \circ f
$$

will be called the composition map.

The composition of two relations can be characterized in terms of the following projection maps. For each of the three pairs $(i, j)$, where $1 \leq i<j \leq 3$, let

$$
\pi_{i j}: X \times X \times X \rightarrow X \times X:\left(x_{1}, x_{2}, x_{3}\right) \mapsto\left(x_{i}, x_{j}\right)
$$

The following lemma is immediate.

Lemma 3.18. If $f$ and $g$ are relations on $X$, then

$$
g \circ f=\pi_{13}\left(\pi_{12}^{-1}(f) \cap \pi_{23}^{-1}(g)\right) .
$$

The next lemma can be checked easily by means of the previous construction.

Lemma 3.19. The composition map preserves inclusion and union, i.e. if $f_{1} \subset$ $f_{2} \subset X \times X$ and $g_{1} \subset g_{2} \subset X \times X$, then $g_{1} \circ f_{1} \subset g_{2} \circ f_{2}$ and

$$
\bigcup \mathcal{G} \circ \bigcup \mathcal{F}=\bigcup\{g \circ f: g \in \mathcal{G} \text { and } f \in \mathcal{F}\}
$$

where $\mathcal{F} \subset \mathcal{R}(X)$ and $\mathcal{G} \subset \mathcal{R}(X)$. 


\section{Relations on topological spaces}

If $X$ is a topological space, then notions of open and closed subsets of $X \times X$ will be extended to relations, using the product topology on $X \times X$. Some of the lemmas of this section can be found in [8].

Definition 4.1. If $X$ is a topological space, then a relation $f$ on $X$ is called open if and only if $f$ is an open subset of $X \times X$. It is called closed if and only if $f$ is a closed subset of $X \times X$.

The operations topological closure and interior also will be extended to relations and will be denoted in the same manner, i.e. if $f$ is a relation on a topological space $X$, then the closure of $f$ is denoted by $\bar{f}$, the interior of $f$ is denoted by $f^{o}$ and the complement of $f$ is denoted by $f^{c}$.

The next lemma is elementary.

Lemma 4.2. If $f$ is a relation on a topological space $X$, then the following statements hold.

(a) $f$ is open if and only if $f^{*}$ is open.

(b) $f$ is closed if and only if $f^{*}$ is closed.

(c) $\overline{f^{*}}=(\bar{f})^{*}$.

The notation $\mathfrak{N}(f)$ will be used to denote the set of all neighborhoods of the relation $f$ in $X \times X$. Similarly, $\mathfrak{N}^{o}(f)$ will denote the set of open neighborhoods and $\overline{\mathfrak{N}}(f)$ will denote the set of closed neighborhoods. Note the convenient fact that a neighborhood of a relation is again a relation.

The next three lemma show how the induced map acts on subsets of $X$.

Lemma 4.3. If $f$ is an open relation on a topological space $X$ and $S \subset X$, then $f(S)$ is open in $X$.

Proof. Let $y \in f(S)$. There exists a point $x \in S$ such that $(x, y) \in f$. By the definition of the product topology and since $f$ is open, there exist open sets $U$ and $V$ such that $(x, y) \in U \times V \subset f$. If $\eta \in V$, then $(x, \eta) \in U \times V \subset f$. Lemma 3.1 therefore implies that $\eta \in f(x) \subset f(S)$, which implies that $V \subset f(S)$ and completes the proof.

Lemma 4.4. If $f$ is a relation on a topological space $X, K$ is a closed subset of $X$ and $U \in \mathfrak{N}^{\circ}(f(K))$, then there exists $\phi \in \mathfrak{N}^{\circ}(f)$ such that $\phi(K) \subset U$.

Proof. Let $\phi \equiv\left(K \times U^{c}\right)^{c}$. Since $f(K) \subset U$, Lemma 3.3 implies that $\left(K \times U^{c}\right) \cap f=$ $\phi^{c} \cap f=\emptyset$ and hence that $f \subset \phi$. Since $\phi$ is open, it is an open neighborhood of $f$. It follows that $\phi^{c} \cap \phi=\left(K \times U^{c}\right) \cap \phi=\emptyset$. Lemma 3.3 therefore implies that $\phi(K) \subset U$, and the proof is complete.

Lemma 4.5. If $f$ is a closed relation on a compact Hausdorff space $X, K$ is a closed subset of $X$ and $U \in \mathfrak{N}(f(K))$, then there exists $V \in \mathfrak{N}(K)$ such that $f(V) \subset U$.

Proof. We can assume $U$ is open. Since $f(K) \subset U$, Lemma 3.3 implies that $\left(K \times U^{c}\right) \cap f=\emptyset$, which means $K \times U^{c} \subset f^{c}$. Now $f^{c}$ is open and $U^{c}$ is closed, hence compact. By a standard result about the product topology, there exist neighborhoods $V$ of $K$ and $W$ of $U^{c}$ such that $V \times W \subset f^{c}$. Then $\left(V \times U^{c}\right) \cap f \subset$ $(V \times W) \cap f=\emptyset$, but then $f(V) \subset U$ by Lemma 3.3 and the proof is complete.

The next lemma shows that neighborhoods behave in a way we expect. 
Lemma 4.6. If $f$ is a relation on a topological space $X$ and $S \subset X$, then the following statements hold.

(a) If $\phi \in \mathfrak{N}^{\circ}(f)$, then $\phi(S) \in \mathfrak{N}^{\circ}(f(S))$.

(b) If $\phi \in \mathfrak{N}(f)$, then $\phi(S) \in \mathfrak{N}(f(S))$.

Proof. First assume that $\phi \in \mathfrak{N}^{\circ}(f)$. Lemma 4.3 implies that $\phi(S)$ is open, while Lemma 3.4 implies that $f(S) \subset \phi(S)$. Therefore, $\phi(S) \in \mathfrak{N}^{\circ}(f(S))$, and property (a) is established.

Now assume that $\phi \in \mathfrak{N}(f)$. There exists an open neighborhood $\psi$ of $f$ such that $\psi \subset \phi$. Property (a) implies that $\psi(S) \in \mathfrak{N}^{\circ}(f(S))$, while Lemma 3.4 implies that $\psi(S) \subset \phi(S)$. Therefore, $\phi(S)$ is a neighborhood of $f(S)$, which establishes property (b) and completes the proof.

The following two theorems will be fundamental later.

Theorem 4.7. If $X$ is a topological space, then the composition map preserves open sets.

Proof. Recall Lemma 3.18 and assume that $f \in \mathcal{R}(X)$ and $g \in \mathcal{R}(X)$ are open. Since the projection maps $\pi_{12}$ and $\pi_{23}$ are continuous, $\pi_{12}^{-1}(f)$ and $\pi_{23}^{-1}(g)$ are open, and so is their intersection. Since the projection $\pi_{13}$ preserves open sets, Lemma 3.18 implies that $g \circ f$ is open and completes the proof.

Theorem 4.8. If $X$ is a compact Hausdorff space, then the composition map is semicontinuous.

Proof. Recall Lemma 3.18 again. Since the maps $\pi_{23}^{-1}$ and $\pi_{12}^{-1}$ preserve inclusion, intersection and closed sets, it follows that the map

$$
\Theta: 2^{X \times X} \times 2^{X \times X} \rightarrow 2^{X \times X \times X}:(f, g) \mapsto \pi_{12}^{-1}(f) \cap \pi_{23}^{-1}(g)
$$

also preserves inclusion, intersection and closed sets. Lemma 2.22 therefore implies that $\Theta$ is semicontinuous. Since $\pi_{13}$ is continuous, Theorem 2.26 implies that the induced map $\pi_{13}: 2^{X \times X \times X} \rightarrow 2^{X \times X}$ is semicontinuous. Lemma 3.18 implies that composition can be written as the composition of semicontinuous maps, that is, $g \circ f=\pi_{13}(\Theta(f, g))$. Lemma 2.20 therefore implies that the map $(f, g) \mapsto g \circ f$ is semicontinuous, and the proof is complete.

Corollary 4.9. If $X$ is a compact Hausdorff space, then the composition map preserves closed sets, i.e. if $f$ and $g$ are closed relations on $X$, then so is $g \circ f$.

We have the following counterpart of Lemma 4.3 for closed relations.

Lemma 4.10. If $f$ is a closed relation on a compact Hausdorff space $X$ and $K \subset X$ is compact, then $f(K)$ is closed.

Proof. Let $y \in f(K)^{c}$. Then $K \times\{y\} \subset f^{c}$. Since $K$ is compact and $f$ is closed, there exists $V \in \mathfrak{N}^{o}(y)$ such that $K \times V \subset f^{c}$, which implies that $V \subset f(K)^{c}$ and we established that $f(K)$ is closed.

The following lemmas will be important later, when we iterate relations. They are built on each other.

Lemma 4.11. If $f$ and $h$ are relations on a set $X$ and $(y, z) \in X \times X$, then

$$
h \circ\{(y, z)\} \circ f=f^{*}(y) \times h(z) .
$$


Lemma 4.12. If $f, g$ and $h$ are relations on a topological space $X$, then $h \circ g \circ f$ is open whenever $f$ and $h$ are open.

Proof. By Lemma 3.19, the composition map preserves union, it follows that for fixed $f$ and $h$, the map

$$
\mathcal{R}(X) \rightarrow \mathcal{R}(X): g \mapsto h \circ g \circ f
$$

also preserves union. Therefore, it suffices to prove that whenever $(y, z) \in g$, $h \circ\{(y, z)\} \circ f$ is open, which, in view of Lemma 4.11, is equivalent to proving that $f^{*}(y) \times h(z)$ is an open subset of $X \times X$. Since $f$ is open, $f^{*}$ is open by Lemma 4.2. Lemma 4.3 implies that both $f^{*}(y)$ and $h(z)$ are open, which implies that $f^{*}(y) \times h(z)$ is open and completes the proof.

Lemma 4.13. If $f, g$ and $h$ are relations on a topological space $X$, then the following properties hold.

(a) If $\phi \in \mathfrak{N}^{\circ}(f)$ and $\psi \in \mathfrak{N}^{\circ}(h)$, then $\psi \circ g \circ \phi \in \mathfrak{N}^{\circ}(h \circ g \circ f)$.

(b) If $\phi \in \mathfrak{N}(f)$ and $\psi \in \mathfrak{N}(h)$, then $\psi \circ g \circ \phi \in \mathfrak{N}(h \circ g \circ f)$.

(c) If $\phi \in \mathfrak{N}(f)$ and $\psi \in \mathfrak{N}(h)$, then $h \circ \bar{g} \circ f \subset \psi \circ g \circ \phi$.

(d) If $f$ and $h$ are open, then $h \circ \bar{g} \circ f=h \circ g \circ f$.

Proof. First assume that $\phi \in \mathfrak{N}^{\circ}(f)$ and $\psi \in \mathfrak{N}^{\circ}(h)$. Lemma 4.12 implies that $\psi \circ g \circ \phi$ is open, while Lemma 3.19 implies that $h \circ g \circ f \subset \psi \circ g \circ \phi$. Therefore, $\psi \circ g \circ \phi$ is an open neighborhood of $h \circ g \circ f$, and property (a) is established.

Now assume that $\phi \in \mathfrak{N}(f)$ and $\psi \in \mathfrak{N}(h)$. There exist relations $\phi^{\prime} \in \mathfrak{N}^{\circ}(f)$ and $\psi^{\prime} \in \mathfrak{N}^{\circ}(h)$ such that $\phi^{\prime} \subset \phi$ and $\psi^{\prime} \subset \psi$. Property (a) implies that $\psi^{\prime} \circ g \circ \phi^{\prime} \in$ $\mathfrak{N}^{\circ}(h \circ g \circ f)$, while Lemma 3.19 implies that $\psi^{\prime} \circ g \circ \phi^{\prime} \subset \psi \circ g \circ \phi$. Therefore, $\psi \circ g \circ \phi$ is a neighborhood of $h \circ g \circ f$, and property (b) is established.

Now let $(x, w) \in h \circ \bar{g} \circ f$. This means there exists a point $(y, z) \in \bar{g}$ such that $(x, y) \in f,(y, z) \in \bar{g}$ and $(z, w) \in h$. Lemma 4.11 implies that $(x, w) \in$ $h \circ\{(y, z)\} \circ f=f^{*}(y) \times h(z)$, which implies that $(y, z) \in f(x) \times h^{*}(w)$. In view of Lemma 3.19, it is enough to establish property (c) for open $\phi$ and $\psi \cdot f \subset \phi$ and $h \subset \psi$, so $(y, z) \in \phi(x) \times \psi^{*}(w)$. This set is open by Lemma 4.3, so there exists $(\eta, \zeta) \in g \cap\left(\phi(x) \times \psi^{*}(w)\right)$, but then $(x, w) \in \phi^{*}(\eta) \times \psi(\zeta)=\psi \circ\{(\eta, \zeta)\} \circ \phi \subset \psi \circ g \circ \phi$ and property (c) is established.

Property (d) is a simple consequence of property (c) and Lemma 3.19.

The last result in this section is the following theorem. It is basically the twovariable version of Theorem 2.25.

Theorem 4.14. If $f$ and $g$ are closed relations on a compact Hausdorff space $X$ and $h \in \mathfrak{N}(g \circ f)$, then there exist $f^{\prime} \in \overline{\mathfrak{N}}(f)$ and $g^{\prime} \in \overline{\mathfrak{N}}(g)$ such that $g^{\prime} \circ f^{\prime} \subset h$.

Proof. Again, let $\pi_{i j}: X \times X \times X \rightarrow X \times X:\left(x_{1}, x_{2}, x_{3}\right) \mapsto\left(x_{i}, x_{j}\right)$. It suffices to assume that $h$ is open. By Lemma $2.24 f=\bigcap \overline{\mathfrak{N}}(f)$ and $g=\bigcap \overline{\mathfrak{N}}(g)$. By Lemma 2.21 it follows that

$$
\begin{aligned}
& \pi_{12}^{-1}(f)=\bigcap_{\phi \in \overline{\mathfrak{N}}(f)} \pi_{12}^{-1}(\phi), \\
& \pi_{23}^{-1}(g)=\bigcap_{\psi \in \overline{\mathfrak{N}}(g)} \pi_{23}^{-1}(\psi) .
\end{aligned}
$$




$$
\begin{aligned}
& \text { Let } \varphi \equiv \pi_{12}^{-1}(f) \cap \pi_{23}^{-1}(g) \text { and } \\
& \qquad \mathfrak{K} \equiv\left\{\pi_{12}^{-1}(\phi) \cap \pi_{23}^{-1}(\psi): \phi \in \overline{\mathfrak{N}}(f) \text { and } \psi \in \overline{\mathfrak{N}}(g)\right\} .
\end{aligned}
$$

Then clearly $\varphi=\bigcap \mathfrak{K}$. Now let

$$
\eta \equiv \pi_{13}^{-1}(h)
$$

Since $h$ is open and $\pi_{13}$ is continuous, it follows that $\eta$ is open. Lemma 3.18 implies that $g \circ f=\pi_{13}(\varphi)$, which, since $g \circ f \subset h$, implies that $\varphi \subset \pi_{13}^{-1}(g \circ f) \subset \pi_{13}^{-1}(h)=\eta$. Therefore, $\eta$ is a neighborhood of $\varphi$. Since any $\phi$ and $\psi$ are closed and since $\pi_{12}$ and $\pi_{23}$ are continuous, each $\pi_{12}^{-1}(\phi) \cap \pi_{23}^{-1}(\psi)$ is closed; hence $\mathfrak{K}$ is a set of closed subsets of $X \times X \times X$. Since $\varphi=\bigcap \mathfrak{K}$, the compactness of $X$ implies the existence of a finite subset of $\mathfrak{K}$ whose intersection is a subset of $\eta$. Therefore,

$$
\bigcap_{k=1}^{n}\left(\pi_{12}^{-1}\left(\phi_{k}\right) \cap \pi_{23}^{-1}\left(\psi_{k}\right)\right) \subset \eta,
$$

where $\phi_{k} \in \overline{\mathfrak{N}}(f)$ and $\psi_{k} \in \overline{\mathfrak{N}}(g)$ for $k=1,2, \ldots, n$.

Now let $f^{\prime} \equiv \bigcap_{k=1}^{n} \phi_{k}$ and $g^{\prime} \equiv \bigcap_{k=1}^{n} \psi_{k}$. Note that $f^{\prime} \in \overline{\mathfrak{N}}(f)$ and $g^{\prime} \in \overline{\mathfrak{N}}(g)$. Furthermore,

$$
\begin{aligned}
& \pi_{12}^{-1}\left(f^{\prime}\right) \cap \pi_{23}^{-1}\left(g^{\prime}\right)=\pi_{12}^{-1}\left(\bigcap_{k=1}^{n} \phi_{k}\right) \cap \pi_{23}^{-1}\left(\bigcap_{j=1}^{n} \psi_{j}\right)= \\
&=\left(\bigcap_{k=1}^{n} \pi_{12}^{-1}\left(\phi_{k}\right)\right) \cap\left(\bigcap_{j=1}^{n} \pi_{23}^{-1}\left(\psi_{j}\right)\right)=\bigcap_{k=1}^{n} \bigcap_{j=1}^{n}\left(\pi_{12}^{-1}\left(\phi_{k}\right) \cap \pi_{23}^{-1}\left(\psi_{j}\right)\right) \subset \\
& \subset \bigcap_{k=1}^{n}\left(\pi_{12}^{-1}\left(\phi_{k}\right) \cap \pi_{23}^{-1}\left(\psi_{k}\right)\right) \subset \eta .
\end{aligned}
$$

Therefore, Lemma 3.18 implies that

$$
g^{\prime} \circ f^{\prime}=\pi_{13}\left(\pi_{12}^{-1}\left(f^{\prime}\right) \cap \pi_{23}^{-1}\left(g^{\prime}\right)\right) \subset \pi_{13}(\eta)=h
$$

and the proof is complete.

\section{ITERATION AND ORBITS}

To define precisely the $n$th iterate of a relation it is convenient to introduce the diagonal (or identity) relation defined as

$$
\iota \equiv\{(x, x) \in X \times X: x \in X\}
$$

This relation has the property that for any relation $f$,

$$
f \circ \iota=\iota \circ f=f \text {. }
$$

Definition 5.1. If $f$ is a relation on a set $X$ and $n$ is a nonnegative integer, then the relation $f^{n}$ is defined inductively by

$$
f^{0}=\iota \text { and } f^{n}=f \circ f^{n-1} \text { for } n=1,2,3, \ldots
$$

A standard argument and Lemma 3.14 (the associativity of composition) imply the next lemma.

Lemma 5.2. If $f$ is a relation on a set $X$ and $n$ and $m$ are nonnegative integers, then $f^{n+m}=f^{n} \circ f^{m}$.

The next lemma also follows by standard induction arguments. Property (a) is implied by Lemma 3.19, while property (b) is implied by Lemma 3.16.

Lemma 5.3. If $f$ and $g$ are relations on a set $X$ and $n$ is a nonnegative integer, then the following properties hold. 
(a) If $g \subset f$, then $g^{n} \subset f^{n}$.

(b) $\left(f^{n}\right)^{*}=\left(f^{*}\right)^{n}$.

Of primary interest in this paper are the relations on compact Hausdorff spaces. We will use the following lemmas later.

Lemma 5.4. If $X$ is a compact Hausdorff space, then $\iota$ is a closed relation.

Lemma 5.5. If $f$ is a closed relation on a compact Hausdorff space $X$ and $g \in$ $\mathfrak{N}(f)$, then there exist $\iota^{\prime}, \iota^{\prime \prime} \in \mathfrak{N}(\iota)$ such that $\iota^{\prime} \circ f \circ \iota^{\prime \prime} \subset g$.

Proof. We will use Theorem 4.14 twice. $g \in \mathfrak{N}(f)=\mathfrak{N}(\iota \circ f)$, so by Theorem 4.14 there exists $f^{\prime} \in \overline{\mathfrak{N}}(f)$ and $\iota^{\prime} \in \overline{\mathfrak{N}}(\iota)$ such that $\iota^{\prime} \circ f^{\prime} \subset g$. Now $f^{\prime} \in \overline{\mathfrak{N}}(f) \subset$ $\mathfrak{N}(f)=\mathfrak{N}(f \circ \iota)$, so by Theorem 4.14 there exists $f^{\prime \prime} \in \overline{\mathfrak{N}}(f)$ and $\iota^{\prime \prime} \in \overline{\mathfrak{N}}(\iota)$ such that $f^{\prime \prime} \circ \iota^{\prime \prime} \subset f^{\prime}$. This means

$$
\iota^{\prime} \circ f \circ \iota^{\prime \prime} \subset \iota^{\prime} \circ f^{\prime \prime} \circ \iota^{\prime \prime} \subset \iota^{\prime} \circ f^{\prime} \subset g
$$

and the proof is complete.

A basic notion in dynamical systems is that of an orbit. For a map, an orbit is the succession of images of a point. For a relation, a point may have no or many image points. An orbit for a relation is one of the possible successions of images.

Definition 5.6. If $f$ is a relation on a set $X$, then an orbit for $f$ is a pair $(p, I)$, where $I$ is an interval of $\mathbb{Z}$, either finite or infinite, and where $p: I \rightarrow X$ satisfies $\left(p_{i}, p_{i+1}\right) \in f$ whenever $i \in I$ and $i+1 \in I$.

We have the following natural connection between iterations of a relation and orbits of a relation. The proof of this theorem can be found in [8].

Theorem 5.7. If $f$ is a relation on a set $X, I$ is an interval of $\mathbb{Z}$ and $p: I \rightarrow X$, then the following statements are equivalent.

(a) $(p, I)$ is an orbit for $f$.

(b) $p_{i+j} \in f^{j}\left(p_{i}\right)$ whenever $j \geq 0, i \in I$, and $i+j \in I$.

A very important notion which requires certain care in case of relations is invariance. In case of a bijective map $f$, a set $S$ is called "invariant" if $f(S)=S$, "forward invariant" if $f(S) \subset S$ and "backward invariant" if $f^{-1}(S) \subset S$. In the case of relations, some confusion develops: for example, the statements $f(S)=S$ and $f^{*}(S)=S$ are identical for bijective maps, but logically independent for relations. The terminology for the different kinds of invariance for relations was developed in a clear and comprehensive way in [8]. The important parts of this terminology for us are the following.

Definition 5.8. If $f$ is a relation on a set $X$ and $S \subset X$, then $S$ is called confining whenever $f(S) \subset S$. It is called invariant whenever $f(S)=S$.

These notions will be utilized later.

\section{The limit Relation $f^{\infty}$}

Our first construction uses only the relation itself, it does not exploit the structure of the underlying space. 
Definition 6.1. If $f$ is a relation on a set $X$, then the limit relation of $f$ is

$$
f^{\infty} \equiv \bigcap_{n \geq 0} \bigcup_{k \geq n} f^{k}
$$

Theorem 6.2. If $f$ and $g$ are relations on a set $X$, then the following properties hold.

(a) If $g \subset f$, then $g^{\infty} \subset f^{\infty}$.

(b) $\left(f^{\infty}\right)^{*}=\left(f^{*}\right)^{\infty}$.

Proof. Let $g \subset f$. Lemma 5.3 implies that $g^{k} \subset f^{k}$ for every nonnegative integer $k$. Therefore,

$$
\bigcup_{k \geq n} g^{k} \subset \bigcup_{k \geq n} f^{k}
$$

for every nonnegative integer $n$, and then Lemma 2.4 implies that

$$
g^{\infty}=\bigcap_{n \geq 0} \bigcup_{k \geq n} g^{k} \subset \bigcap_{n \geq 0} \bigcup_{k \geq n} f^{k}=f^{\infty}
$$

and establishes property (a).

Lemmas 3.11 and 5.3 imply that

$$
\begin{aligned}
\left(f^{\infty}\right)^{*} & =\left(\bigcap_{n \geq 0} \bigcup_{k \geq n} f^{k}\right)^{*}=\bigcap_{n \geq 0}\left(\bigcup_{k \geq n} f^{k}\right)^{*}= \\
& =\bigcap_{n \geq 0} \bigcup_{k \geq n}\left(f^{k}\right)^{*}=\bigcap_{n \geq 0} \bigcup_{k \geq n}\left(f^{*}\right)^{k}=\left(f^{*}\right)^{\infty},
\end{aligned}
$$

which establishes property (b) and completes the proof.

Theorem 6.3. If $f$ is a relation on a set $X$, then the following inclusions hold.

(a) $f \circ f^{\infty} \subset f^{\infty}$.

(b) $f^{\infty} \circ f \subset f^{\infty}$.

(c) $f^{\infty} \circ f^{\infty} \subset f^{\infty}$.

Proof. Lemma 3.19 implies that for any relations $f$ and $g$,

$$
g \circ\left(\bigcap_{n \geq 0} \bigcup_{k \geq n} f^{k}\right) \subset \bigcap_{n \geq 0}\left(g \circ \bigcup_{k \geq n} f^{k}\right)=\bigcap_{n \geq 0} \bigcup_{k \geq n} g \circ f^{k},
$$

which implies that

$$
g \circ f^{\infty} \subset \bigcap_{n \geq 0} \bigcup_{k \geq n} g \circ f^{k}
$$

In particular,

$$
f \circ f^{\infty} \subset \bigcap_{n \geq 0} \bigcup_{k \geq n} f \circ f^{k}=\bigcap_{n \geq 0} \bigcup_{k \geq n} f^{k+1}=f^{\infty},
$$

which establishes inclusion (a).

Now let $h \equiv f^{*}$. Theorem 6.2, Lemma 3.16, inclusion (a) of this theorem and Lemma 3.10 imply that

$$
\left(h^{*}\right)^{\infty} \circ h^{*}=\left(h^{\infty}\right)^{*} \circ h^{*}=\left(h \circ h^{\infty}\right)^{*} \subset\left(h^{\infty}\right)^{*}=\left(h^{*}\right)^{\infty},
$$

which, since Lemma 3.10 implies that $h^{*}=f$, establishes inclusion (b).

A simple induction argument applied to inclusion (b) of this theorem produces the inclusion

$$
f^{\infty} \circ f^{k} \subset f^{\infty}
$$


for all nonnegative integers $k$. For $g=f^{\infty}$, inclusion (2) becomes

$$
f^{\infty} \circ f^{\infty} \subset \bigcap_{n \geq 0} \bigcup_{k \geq n} f^{\infty} \circ f^{k} \subset \bigcap_{n \geq 0} \bigcup_{k \geq n} f^{\infty}=f^{\infty} .
$$

which establishes inclusion (c) and completes the proof.

Remark 6.4. It is not necessarily true, that $f \circ f^{\infty}=f^{\infty}, f^{\infty} \circ f=f^{\infty}$ or $f^{\infty} \circ f^{\infty}=f^{\infty}$. Let $X \equiv[0,1]$ and

$$
\begin{gathered}
f \equiv\left\{(1, x) \in X \times X: 0 \leq x \leq \frac{2}{3}\right\} \cup \\
\cup\left\{(x, y) \in X \times X: y=2 x-\frac{2}{3}, \text { where } \frac{1}{3} \leq x \leq \frac{2}{3}\right\} .
\end{gathered}
$$

It is easy to check that

$$
f^{\infty}=\left\{(1, x) \in X \times X: 0 \leq x \leq \frac{2}{3}\right\} \cup\left\{\left(\frac{2}{3}, \frac{2}{3}\right)\right\} .
$$

Clearly $(1,0) \in f^{\infty}$, but $(1,0) \notin f^{\infty} \circ f$ and $(1,0) \notin f^{\infty} \circ f^{\infty}$. Also, $g \circ g^{\infty} \neq g^{\infty}$ for $g \equiv f^{*}$.

\section{The $\omega$-Limit RELATION $f^{\omega}$}

Our second construction will be similar to the first one, but here we will use the topology of the space the relation is defined on.

Definition 7.1. If $f$ is a relation on a topological space $X$, then the $\omega$-limit relation of $f$ is

$$
f^{\omega} \equiv \bigcap_{n \geq 0} \overline{\bigcup_{k \geq n} f^{k}}
$$

Theorem 7.2. If $f$ and $g$ are relations on a topological space $X$, then the following properties hold.
(a) $f^{\infty} \subset f^{\omega}$.
(b) If $g \subset f$, then $g^{\omega} \subset f^{\omega}$.
(c) $\left(f^{\omega}\right)^{*}=\left(f^{*}\right)^{\omega}$.
(d) $f^{\omega}$ is closed.

Proof. Property (a) follows directly from the definitions, while property (b) follows from the definition and from Lemma 5.3. Property (d) also follows from the definition, since the intersection of closed sets is closed. Lemmas 3.11, 4.2, and 5.3 imply that

$$
\begin{aligned}
\left(f^{\omega}\right)^{*} & \left.=\left(\bigcap_{n \geq 0} \overline{\bigcup_{k \geq n} f^{k}}\right)^{*}=\bigcap_{n \geq 0} \overline{\left(\bigcup_{k \geq n} f^{k}\right.}\right)^{*}=\bigcap_{n \geq 0} \overline{\left(\bigcup_{k \geq n} f^{k}\right)^{*}}= \\
& =\bigcap_{n \geq 0} \overline{\bigcup_{k \geq n}\left(f^{k}\right)^{*}}=\bigcap_{n \geq 0} \overline{\bigcup_{k \geq n}\left(f^{*}\right)^{k}}=\left(f^{*}\right)^{\omega},
\end{aligned}
$$

which establishes property (c) and completes the proof.

Compare the next theorem to Theorem 6.3.

Theorem 7.3. If $f$ is a closed relation on a compact Hausdorff space $X$, then the following inclusions hold.
(a) $f \circ f^{\omega} \supset f^{\omega}$.
(b) $f^{\omega} \circ f \supset f^{\omega}$. 
(c) $f^{\omega} \circ f^{\omega} \supset f^{\omega}$.

Proof. Let $f$ and $g$ be closed relations on a compact Hausdorff space $X$. Note that $\{g\}$ and $\left\{\overline{\bigcup_{k \geq n} f^{k}}: n \geq 0\right\}$ are both directed families of closed subsets of $X \times X$. Since the composition map is semicontinuous, it follows that

$$
g \circ f^{\omega}=g \circ \bigcap_{n \geq 0} \overline{\bigcup_{k \geq n} f^{k}}=\bigcap_{n \geq 0}\left(g \circ \overline{\bigcup_{k \geq n} f^{k}}\right) .
$$

Since the composition map preserves union, inclusion and closed sets, it follows that for each nonnegative integer $n$,

$$
\overline{\bigcup_{k \geq n} g \circ f^{k}}=\overline{g \circ \bigcup_{k \geq n} f^{k}} \subset \bar{g} \circ \overline{\bigcup_{k \geq n} f^{k}}=g \circ \overline{\bigcup_{k \geq n} f^{k}},
$$

which, with equality (3) and by Lemma 2.4, implies that

$$
\bigcap_{n \geq 0} \overline{\bigcup_{k \geq n} g \circ f^{k}} \subset g \circ f^{\omega} .
$$

For $g=f$ this inclusion becomes

$$
f^{\omega}=\bigcap_{n \geq 0} \overline{\bigcup_{k \geq n} f^{k+1}}=\bigcap_{n \geq 0} \overline{\bigcup_{k \geq n} f \circ f^{k}} \subset f \circ f^{\omega},
$$

which establishes inclusion (a).

Now let $h \equiv f^{*}$. Theorem 7.2, Lemma 3.16, inclusion (a) of this theorem and Lemma 3.10 imply that

$$
\left(h^{*}\right)^{\omega}=\left(h^{\omega}\right)^{*} \subset\left(h \circ h^{\omega}\right)^{*}=\left(h^{\omega}\right)^{*} \circ h^{*}=\left(h^{*}\right)^{\omega} \circ h^{*},
$$

which, since Lemma 3.10 implies that $h^{*}=f$, establishes inclusion (b).

A simple induction argument applied to inclusion (b) of this theorem produces the inclusion

$$
f^{\omega} \subset f^{\omega} \circ f^{k}
$$

for all nonnegative integers $k$. For $g=f^{\omega}$, inclusion (4) becomes

$$
f^{\omega}=\bigcap_{n \geq 0} \overline{\bigcup_{k \geq n} f^{\omega}} \subset \bigcap_{n \geq 0} \overline{\bigcup_{k \geq n} f^{\omega} \circ f^{k}} \subset f^{\omega} \circ f^{\omega}
$$

which establishes inclusion (c) and completes the proof.

Remark 7.4. It is not necessarily true, that $f \circ f^{\omega}=f^{\omega}, f^{\omega} \circ f=f^{\omega}$ or $f^{\omega} \circ f^{\omega}=$ $f^{\omega}$. Let $X \equiv[0,1]$ and

$$
f \equiv\{(0,1)\} \cup\{(x, y) \in X \times X: x=2 y\} .
$$

Also, let $S \equiv\left\{1 / 2^{n}: n=0,1,2, \ldots\right\} \cup\{0\} \subset X$. It is easy to check that

$$
f^{\omega}=\{(0, x): x \in S\} \cup\{(x, 0): x \in X\} .
$$

Clearly $(1,1 / 2) \notin f^{\omega}$, but $(1,1 / 2) \in f \circ f^{\omega},(1,1 / 2) \in f^{\omega} \circ f$ and $(1,1 / 2) \in f^{\omega} \circ f^{\omega}$.

The next lemma gives a nontrivial connection between the limit relation and the $\omega$-limit relation.

Lemma 7.5. If $f$ is a closed relation on a compact Hausdorff space $X$ and $g \in$ $\mathfrak{N}(f)$, then $g^{\infty} \in \mathfrak{N}\left(f^{\omega}\right)$. 
Proof. In view of Theorem 6.2, it suffices to establish the conclusion under the assumption that $g$ is open. Lemma 5.5 implies the existence of relations $\iota^{\prime}, \iota^{\prime \prime} \in \mathfrak{N}(\iota)$ such that $\iota^{\prime} \circ f \circ \iota^{\prime \prime} \subset g$. Lemma 3.19 implies that $\iota=\iota \circ \iota \subset \iota^{\prime \prime} \circ \iota^{\prime}$. A simple induction argument therefore establishes that

$$
\iota^{\prime} \circ f^{k} \circ \iota^{\prime \prime} \subset g^{k}
$$

for all positive $k$. Lemmas 4.13 and 3.19 therefore imply that

$$
\overline{\bigcup_{k \geq n} f^{k}} \subset \iota^{\prime} \circ\left(\bigcup_{k \geq n} f^{k}\right) \circ \iota^{\prime \prime}=\bigcup_{k \geq n} \iota^{\prime} \circ f^{k} \circ \iota^{\prime \prime} \subset \bigcup_{k \geq n} g^{k},
$$

for every nonnegative integer $n$. By Lemma 2.4 it follows that

$$
f^{\omega} \subset g^{\infty} \text {. }
$$

This inclusion, combined with inclusions (a) and (b) of Theorem 7.3, Lemma 3.19 and inclusions (a) and (b) of Theorem 6.3 implies that

$$
f^{\omega} \subset f \circ f^{\omega} \circ f \subset g \circ g^{\infty} \circ g \subset g^{\infty} .
$$

Since $g$ is open by assumption, Lemma 4.12 implies that $g \circ g^{\infty} \circ g$ is open, which implies that $g^{\infty} \in \mathfrak{N}\left(f^{\omega}\right)$, and the lemma is proved.

\section{The Conley Relation $f^{\Omega}$}

Our third construction is the most important. It uses the first construction and exploits how the relation "sits" in the topological space $X \times X$ via the neighborhood structure.

Definition 8.1. If $f$ is a relation on a topological space $X$, then the Conley relation of $f$ is

$$
f^{\Omega} \equiv \bigcap_{\phi \in \overline{\mathfrak{N}}(f)} \phi^{\infty} .
$$

Theorem 8.2. If $f$ and $g$ are relations on a topological space $X$, then the following properties hold.
(a) $f^{\infty} \subset f^{\Omega}$.
(b) If $g \subset f$, then $g^{\Omega} \subset f^{\Omega}$.
(c) $\left(f^{\Omega}\right)^{*}=\left(f^{*}\right)^{\Omega}$.

Proof. Theorem 6.2 implies that $f^{\infty} \subset \phi^{\infty}$ whenever $f \subset \phi$. Therefore,

$$
f^{\infty} \subset \bigcap_{\phi \in \overline{\mathfrak{N}}(f)} \phi^{\infty}=f^{\Omega},
$$

which establishes property (a).

Let $\phi \in \overline{\mathfrak{N}}(f) . g \subset f$ implies that $\phi \in \overline{\mathfrak{N}}(g)$, and then Theorem 6.2 together with Lemma 2.4 implies that

$$
g^{\Omega}=\bigcap_{\psi \in \overline{\mathfrak{N}}(g)} \psi^{\infty} \subset \bigcap_{\phi \in \overline{\mathfrak{N}}(f)} \phi^{\infty}=f^{\Omega},
$$

which establishes property (b).

Note that Lemmas 3.10 and 4.2 imply that $\psi \in \overline{\mathfrak{N}}\left(f^{*}\right)$ if and only if $\psi^{*} \in \overline{\mathfrak{N}}(f)$, which implies that

$$
\left(f^{*}\right)^{\Omega}=\bigcap_{\psi \in \overline{\mathfrak{N}}\left(f^{*}\right)} \psi^{\infty}=\bigcap_{\psi^{*} \in \overline{\mathfrak{N}}(f)} \psi^{\infty} .
$$


Lemma 3.11, Theorem 6.2 and Lemma 3.10 therefore imply that

$$
\begin{aligned}
\left(f^{\Omega}\right)^{*} & =\left(\bigcap_{\phi \in \overline{\mathfrak{N}}(f)} \phi^{\infty}\right)^{*}=\bigcap_{\phi \in \overline{\mathfrak{N}}(f)}\left(\phi^{\infty}\right)^{*}= \\
& =\bigcap_{\phi \in \overline{\mathfrak{N}}(f)}\left(\phi^{*}\right)^{\infty}=\bigcap_{\psi^{*} \in \overline{\mathfrak{N}}(f)} \psi^{\infty}=\left(f^{*}\right)^{\Omega},
\end{aligned}
$$

which establishes property (c) and completes the proof.

The next theorem shows that $f^{\Omega}$ is "maximal" in the sense that we do not get more information if we replace $\phi^{\infty}$ with $\phi^{\omega}$ or with $\phi^{\Omega}$ in the construction.

Theorem 8.3. If $f$ is a closed relation on a compact Hausdorff space $X$, then the following properties hold.

(a) $f^{\omega} \subset f^{\Omega}$.

(b) $f^{\Omega}=\bigcap\left\{\phi^{\Omega}: \phi \in \overline{\mathfrak{N}}(f)\right\}$.

(c) $f^{\Omega}=\bigcap\left\{\phi^{\omega}: \phi \in \overline{\mathfrak{N}}(f)\right\}$.

(d) $f^{\Omega}$ is closed.

Proof. Lemma 7.5 implies that $f^{\omega} \subset \phi^{\infty}$ whenever $\phi \in \overline{\mathfrak{N}}(f)$. Therefore,

$$
f^{\omega} \subset \bigcap_{\phi \in \overline{\mathfrak{N}}(f)} \phi^{\infty}=f^{\Omega},
$$

and property (a) is established.

Theorem 8.2 implies that $f^{\Omega} \subset \phi^{\Omega}$ whenever $\phi \in \overline{\mathfrak{N}}(f)$. Therefore,

$$
f^{\Omega} \subset \bigcap_{\phi \in \overline{\mathfrak{N}}(f)} \phi^{\Omega} .
$$

Now let $\psi \in \overline{\mathfrak{N}}(f)$. The normality of the space $X \times X$ implies the existence of a relation $\phi \in \overline{\mathfrak{N}}(f)$ such that $\psi \in \overline{\mathfrak{N}}(\phi)$. The definition of $\phi^{\Omega}$ implies that $\phi^{\Omega} \subset \psi^{\infty}$. Lemma 2.4 therefore implies that

$$
\bigcap_{\phi \in \overline{\mathfrak{N}}(f)} \phi^{\Omega} \subset \bigcap_{\psi \in \overline{\mathfrak{N}}(f)} \psi^{\infty}=f^{\Omega},
$$

which, with inclusion (5), establishes property (b).

Next note that Theorem 7.2 and property (a) of this theorem imply that $\phi^{\infty} \subset$ $\phi^{\omega} \subset \phi^{\Omega}$ for every $\phi \in \overline{\mathfrak{N}}(f)$. Property (b) of this theorem therefore implies that

$$
f^{\Omega}=\bigcap_{\phi \in \overline{\mathfrak{N}}(f)} \phi^{\infty} \subset \bigcap_{\phi \in \overline{\mathfrak{N}}(f)} \phi^{\omega} \subset \bigcap_{\phi \in \overline{\mathfrak{N}}(f)} \phi^{\Omega}=f^{\Omega},
$$

which establishes property (c).

Finally, property (c) of this theorem implies that $f^{\Omega}$ can be written as the intersection of closed sets and thus is itself closed. Property (d) is established, and the proof is complete.

Corollary 8.4. If $f$ is a closed relation on a compact Hausdorff space $X$ and $K$ is a closed subset of $X$, then $f^{\Omega}(K)$ is closed.

Proof. Theorem 8.3 implies that $f^{\Omega}$ is closed, so Lemma 4.10 implies that $f^{\Omega}(K)$ is closed. 
Compare the next theorem to Theorems 6.3 and 7.3.

Theorem 8.5. If $f$ is a closed relation on a compact Hausdorff space $X$, then the following properties hold.
(a) $f \circ f^{\Omega}=f^{\Omega}$.
(b) $f^{\Omega} \circ f=f^{\Omega}$.
(c) $f^{\Omega} \circ f^{\Omega}=f^{\Omega}$.

Proof. Since composition preserves union, inclusions (a) and (c) from Theorem 6.3 imply that

$$
\left(\phi \cup \phi^{\infty}\right) \circ \phi^{\infty}=\left(\phi \circ \phi^{\infty}\right) \cup\left(\phi^{\infty} \circ \phi^{\infty}\right) \subset \phi^{\infty} .
$$

Since composition preserves inclusion, if $\phi \in \overline{\mathfrak{N}}(f)$, then

$$
\left(f \circ f^{\Omega}\right) \cup\left(f^{\Omega} \circ f^{\Omega}\right)=\left(f \cup f^{\Omega}\right) \circ f^{\Omega} \subset\left(\phi \cup \phi^{\infty}\right) \circ \phi^{\infty} \subset \phi^{\infty},
$$

which implies that

$$
\left(f \circ f^{\Omega}\right) \cup\left(f^{\Omega} \circ f^{\Omega}\right) \subset f^{\Omega} .
$$

Theorem 7.2 implies that $\left\{\phi^{\omega}: \phi \in \overline{\mathfrak{N}}(f)\right\}$ is a directed family of closed relations. Since $\{\phi: \phi \in \overline{\mathfrak{N}}(f)\}$ is also a directed family of closed relations and since composition is semicontinuous by Theorem 4.8, it follows (with the aid of Theorem 8.3 and Lemma 2.24) that for any closed relation $f$,

$$
f \circ f^{\Omega}=\left(\bigcap_{\psi \in \overline{\mathfrak{N}}(f)} \psi\right) \circ\left(\bigcap_{\phi \in \overline{\mathfrak{N}}(f)} \phi^{\omega}\right)=\bigcap_{\psi \in \overline{\mathfrak{N}}(f)} \bigcap_{\phi \in \overline{\mathfrak{N}}(f)} \psi \circ \phi^{\omega}
$$

and

$$
f^{\Omega} \circ f^{\Omega}=\left(\bigcap_{\psi \in \overline{\mathfrak{N}}(f)} \psi^{\omega}\right) \circ\left(\bigcap_{\phi \in \overline{\mathfrak{N}}(f)} \phi^{\omega}\right)=\bigcap_{\psi \in \overline{\mathfrak{N}}(f)} \bigcap_{\phi \in \overline{\mathfrak{N}}(f)} \psi^{\omega} \circ \phi^{\omega} .
$$

Since $\psi \in \overline{\mathfrak{N}}(f)$ and $\phi \in \overline{\mathfrak{N}}(f)$ implies that $\psi \cap \phi \in \overline{\mathfrak{N}}(f)$, it follows that

$$
f \circ f^{\Omega}=\bigcap_{\phi \in \overline{\mathfrak{N}}(f)} \phi \circ \phi^{\omega}
$$

and

$$
f^{\Omega} \circ f^{\Omega}=\bigcap_{\phi \in \overline{\mathfrak{N}}(f)} \phi^{\omega} \circ \phi^{\omega} .
$$

Inclusions (a) and (c) of Theorem 7.3 and Theorem 8.3 therefore imply that

$$
f^{\Omega}=\bigcap_{\phi \in \overline{\mathfrak{N}}(f)} \phi^{\omega} \subset\left(f \circ f^{\Omega}\right) \cap\left(f^{\Omega} \circ f^{\Omega}\right) .
$$

This inclusion, together with inclusion (6), produces

$$
f^{\Omega} \subset\left(f \circ f^{\Omega}\right) \cap\left(f^{\Omega} \circ f^{\Omega}\right) \subset\left(f \circ f^{\Omega}\right) \cup\left(f^{\Omega} \circ f^{\Omega}\right) \subset f^{\Omega},
$$

which implies that

$$
f \circ f^{\Omega}=f^{\Omega} \circ f^{\Omega}=f^{\Omega}
$$

and establishes properties (a) and (c).

Now let $h \equiv f^{*}$. Theorem 8.2, Lemma 3.16, property (a) of this theorem and Lemma 3.10 imply that

$$
\left(h^{*}\right)^{\Omega} \circ h^{*}=\left(h^{\Omega}\right)^{*} \circ h^{*}=\left(h \circ h^{\Omega}\right)^{*}=\left(h^{\Omega}\right)^{*}=\left(h^{*}\right)^{\Omega},
$$

which, since Lemma 3.10 implies that $h^{*}=f$, establishes property (b) and completes the proof. 
We will use the following lemmas later. They describe how the Conley relation behaves for "close" relations.

Lemma 8.6. If $f$ is a closed relation on a compact Hausdorff space $X$ and $g \in$ $\overline{\mathfrak{N}}(f)$, then $g^{\Omega} \in \overline{\mathfrak{N}}\left(f^{\Omega}\right)$.

Proof. The normality of the space $X \times X$ implies that there exists $h \in \overline{\mathfrak{N}}(f)$, such that $g \in \overline{\mathfrak{N}}(h)$. Now from Lemma 7.5 we know that $g^{\infty} \in \mathfrak{N}\left(h^{\omega}\right)$, so there exists an open relation $\xi$ such that $h^{\omega} \subset \xi \subset g^{\infty}$. But then

$$
f^{\Omega}=\bigcap_{\phi \in \overline{\mathfrak{N}}(f)} \phi^{\infty} \subset h^{\infty} \subset h^{\omega} \subset \xi \subset g^{\infty} \subset g^{\Omega},
$$

which proves the claim.

Lemma 8.7. If $f$ is a closed relation on a compact Hausdorff space $X$ and $(x, y) \notin$ $f^{\Omega}$, then there exists $g \in \overline{\mathfrak{N}}(f)$ such that $(x, y) \notin g^{\Omega}$.

Proof. By the definition of $f^{\Omega},(x, y) \notin f^{\Omega}$ if and only if there exists $\phi \in \overline{\mathfrak{N}}(f)$ such that $(x, y) \notin \phi^{\infty}$. By the normality of the space $X \times X$ there exists $g \in \overline{\mathfrak{N}}(f)$, such that $\phi \in \overline{\mathfrak{N}}(g)$. But then

$$
g^{\Omega}=\bigcap_{\psi \in \overline{\mathfrak{N}}(g)} \psi^{\infty} \subset \phi^{\infty},
$$

which implies $(x, y) \notin g^{\Omega}$ and proves the claim.

The next theorem shows that the Conley relation is semicontinuous on compact Hausdorff spaces.

Theorem 8.8. If $X$ is a compact Hausdorff space, then the map ${ }^{\Omega}: 2^{X \times X} \rightarrow$ $2^{X \times X}, f \mapsto f^{\Omega}$ is semicontinuous.

Proof. ${ }^{\Omega}$ preserves inclusion and closed sets by Theorems 8.2 and 8.3 , so by Theorem 2.25 we only have to prove that if $\psi \in \overline{\mathfrak{N}}\left(f^{\Omega}\right)$, then there exists $g \in \overline{\mathfrak{N}}(f)$ such that $g^{\Omega} \subset \psi$. There exists $\phi \in \mathfrak{N}^{o}\left(f^{\Omega}\right)$ such that $\phi \subset \psi$. For every $(x, y) \in \phi^{c}$ $(x, y) \notin f^{\Omega}$, and by Lemma 8.7 there exists $g_{(x, y)} \in \overline{\mathfrak{N}}(f)$ such that $(x, y) \notin g_{(x, y)}^{\Omega}$, which implies $(x, y) \in\left(g_{(x, y)}^{\Omega}\right)^{c}$. This way we obtained an open cover of the compact set $\phi^{c}$, so there exist finitely many $\left(g_{\left(x_{i}, y_{i}\right)}^{\Omega}\right)^{c}, i=1,2, \ldots n$ such that

$$
\phi^{c} \subset \bigcup_{i=1}^{n}\left(g_{\left(x_{i}, y_{i}\right)}^{\Omega}\right)^{c} .
$$

Let $g \equiv \bigcap_{i=1}^{n} g_{\left(x_{i}, y_{i}\right)} \cdot g$ is clearly a closed neighborhood of $f$ and by Theorem 8.2

$$
g^{\Omega}=\left(\bigcap_{i=1}^{n} g_{\left(x_{i}, y_{i}\right)}\right)^{\Omega} \subset \bigcap_{i=1}^{n} g_{\left(x_{i}, y_{i}\right)}^{\Omega} \subset \phi \subset \psi
$$

and the proof is complete.

The last theorem of this section gives the result that the Conley relation of a Conley relation is itself.

Theorem 8.9. If $f$ is a closed relation on a compact Hausdorff space $X$, then

$$
\left(f^{\Omega}\right)^{\Omega}=f^{\Omega}
$$


Proof. If $\psi$ is a closed relation on $X$, then by Theorem 8.5 and an induction argument $\left(\psi^{\Omega}\right)^{k}=\psi^{\Omega}$ for $k=1,2, \ldots$. Then by the definition of the limit relation,

$$
\left(\psi^{\Omega}\right)^{\infty}=\bigcap_{n \geq 0} \bigcup_{k \geq n}\left(\psi^{\Omega}\right)^{k}=\bigcap_{n \geq 1} \psi^{\Omega}=\psi^{\Omega} .
$$

By Theorem 8.2 we get immediately that

$$
f^{\Omega}=\left(f^{\Omega}\right)^{\infty} \subset\left(f^{\Omega}\right)^{\Omega} .
$$

Suppose now that $(x, y) \notin f^{\Omega}$. By Theorem 8.3 this means there exists $\phi \in \overline{\mathfrak{N}}(f)$ such that $(x, y) \notin \phi^{\Omega}$. This implies that $(x, y) \notin\left(\phi^{\Omega}\right)^{\infty}=\phi^{\Omega}$, but by Lemma 8.6 $\phi^{\Omega} \in \overline{\mathfrak{N}}\left(f^{\Omega}\right)$, so there exists a closed neighborhood of $f^{\Omega}$ such that $(x, y)$ is not in the limit relation of that neighborhood, which means $(x, y) \notin\left(f^{\Omega}\right)^{\Omega}$ and this implies

$$
\left(f^{\Omega}\right)^{\Omega} \subset f^{\Omega}
$$

which proves the claim.

\section{Recurrence}

In this section, we generalize the well-known notions of periodic point, nonwandering set and chain recurrent set to dynamical systems generated by iterating relations. These notions will be natural extensions of the already known ones. We will find a connection between these recurrent sets and the previously defined relations $f^{\infty}, f^{\omega}$ and $f^{\Omega}$. At the end of the section, we will define an interesting equivalence relation.

First we define the simplest case of recurrence.

Definition 9.1. If $f$ is a relation on a set $X$, then a point $x \in X$ is called a fixed point for $f$ if $(x, x) \in f$. The set of all fixed points will be denoted

$$
\mathrm{F}(f) \equiv\{x \in X:(x, x) \in f\} .
$$

This definition is clearly consistent with the definition of fixed points in the case of a map on a set $X ;(x, x) \in f$ if and only if $x \in f(x)$, in the case of a map $x \in f(x)$ if and only if $x=f(x)$.

The following lemmas are elementary; we omit the proofs.

Lemma 9.2. If $f$ is a relation on a set $X$, then

$$
\mathrm{F}(f)=\pi_{1}(f \cap \iota),
$$

where $\pi_{1}: X \times X \rightarrow X:(x, y) \mapsto x$ is the standard projection map.

Lemma 9.3. If $g$ is a closed relation on a compact Hausdorff space $X$, then $\pi_{1}(g)$ is a closed subset of $X$.

We will use the next lemma later.

Lemma 9.4. If $f$ is a closed relation on a compact Hausdorff space $X$, then $\mathrm{F}(f)$ is a closed subset of $X$.

Proof. This is a simple consequence of Lemmas 5.4, 9.3 and 9.2.

A straightforward induction argument establishes inclusion (a) of the following lemma. Inclusion (b) is an immediate consequence. 
Lemma 9.5. If $f$ is a relation on a set $X$ and $q$ and $n$ are positive integers, then the following inclusions hold.

(a) $\mathrm{F}(f) \subset \mathrm{F}\left(f^{n}\right)$.

(b) $\mathrm{F}\left(f^{q}\right) \subset \mathrm{F}\left(f^{n q}\right)$.

The next lemma follows directly from the definitions.

Lemma 9.6. If $\mathcal{F} \subset \mathcal{R}(X)$, then

$$
\mathrm{F}(\bigcap \mathcal{F})=\bigcap_{f \in \mathcal{F}} \mathrm{F}(f) .
$$

Now we define the next simplest case of recurrence: periodicity.

Definition 9.7. If $f$ is a relation on a set $X$ and $q$ is a positive integer, then $x \in X$ is called a periodic point for $f$ of period $q$ if $x$ is a fixed point for $f^{q}$. The set of periodic points of period $q$ will be denoted

$$
\mathrm{P}^{q}(f) \equiv \mathrm{F}\left(f^{q}\right) .
$$

The set of periodic points of arbitrary period will be denoted

$$
\mathrm{P}(f) \equiv \bigcup_{q \geq 1} \mathrm{P}^{q}(f)
$$

The definition is clearly consistent again with the definition of periodic points for maps on a set $X ;(x, x) \in f^{q}$ if and only if $x \in f^{q}(x)$, in the case of a map $x \in f^{q}(x)$ if and only if $x=f^{q}(x)$. If $f$ is a relation on a set $X$ and $x$ is a periodic point of period $q$, Theorem 5.7 implies the existence of an orbit $(p,(-\infty, \infty))$ such that $p_{k q}=x$ and $p_{t}=p_{s}$ if $t \equiv s(\bmod q)$, for all $k \in \mathbb{Z}$.

The following theorem characterizes the periodic points of a relation as the fixed points of the relation $f^{\infty}$.

Theorem 9.8. If $f$ is a relation on a set $X$, then

$$
\mathrm{P}(f)=\mathrm{F}\left(f^{\infty}\right) .
$$

Proof. Let $x \in \mathrm{F}\left(f^{\infty}\right)$. Since $(x, x) \in \bigcup_{k \geq 1} f^{k}$, there exists a positive integer $q$ such that $(x, x) \in f^{q}$. Therefore, $x \in \mathrm{F}\left(f^{q}\right)=\mathrm{P}^{q}(f) \subset \mathrm{P}(f)$, which implies that

$$
\mathrm{F}\left(f^{\infty}\right) \subset \mathrm{P}(f)
$$

Now let $x \in \mathrm{P}(f)$, and let $n$ be a positive integer. Note that $x \in \mathrm{P}^{q}(f)$ for some $q \geq 1$, and choose a positive integer $j$ such that $j q \geq n$. Lemma 9.5 implies that $x \in \mathrm{F}\left(f^{q}\right) \subset \mathrm{F}\left(f^{j q}\right)$, which implies that $(x, x) \in f^{j q} \subset \bigcup_{k>n} f^{k}$. Since $n$ is arbitrary, $(x, x) \in \bigcap_{n \geq 0} \bigcup_{k \geq n} f^{k}=f^{\infty}$. Therefore, $x \in \mathrm{F}\left(f^{\infty}\right)$, which implies that

$$
\mathrm{P}(f) \subset \mathrm{F}\left(f^{\infty}\right)
$$

and completes the proof.

We see from here that periodicity is a "topology-independent" property: in the constructions, we did not use the properties of the space $X$.

The next part will concern the nonwandering set. In order to give motivation to this part, we define in two easy steps the nonwandering set for flows. We follow the treatment of Conley [3]. The first step is the definition of the $\omega$-limit set of a set. 
Definition 9.9. If $\varphi^{t}$ is a flow on a topological space $X$, then the $\omega$-limit set of a set $U \subset X$ is

$$
\omega(U) \equiv \bigcap_{t \geq 0} \overline{\varphi^{[t, \infty)}(U)} .
$$

The second step is the actual definition of the nonwandering set.

Definition 9.10. If $\varphi^{t}$ is a flow on a topological space $X$, then $x \in X$ is called nonwandering for $\varphi^{t}$ if $x \in \omega(U)$ for every neighborhood $U$ of $x$ in $X$. The set of nonwandering points is usually denoted by $\mathrm{N}_{\omega}\left(\varphi^{t}\right)$.

Now we will try to generalize this notion to relations. $\omega$-limit sets of sets for relations were generalized by McGehee [8]. One of these generalizations is the so called "strict $\omega$-limit set". It is easy to see, that this is the discrete analogy of the $\omega$-limit set of a set for flows.

Definition 9.11. If $f$ is a relation on a topological space $X$, then the strict $\omega$-limit set of a set $U \subset X$ is

$$
\hat{\omega}(U) \equiv \bigcap_{n \geq 0} \overline{\bigcup_{k \geq n} f^{k}(U)} .
$$

Remark 9.12. In the same article [8], McGehee gave another generalization of the $\omega$-limit set of a set for relations. We will give that definition later, when we will use it in the study of attractors and repellers. In the special case of maps, the two generalizations produce the same sets.

Now the definition of the nonwandering set is immediate.

Definition 9.13. If $f$ is a closed relation on a compact Hausdorff space $X$, then $x \in X$ is called nonwandering for $f$ if $x \in \hat{\omega}(U)$ for every neighborhood $U$ of $x$ in $X$. The set of nonwandering points will be denoted by $\mathrm{N}_{\omega}(f)$.

In order to prove the main result, the characterization of this set with the aid of $f^{\omega}$, we will need the following lemmas.

Lemma 9.14. If $g$ is a relation on a compact Hausdorff space $X$ and $U \subset X$ is closed, then

$$
\overline{g(U)} \subset \bar{g}(U) .
$$

Proof. This is an immediate consequence of Lemma 4.10.

Lemma 9.15. If $f$ is a closed relation on a compact Hausdorff space $X$ and $U \subset X$ is closed, then

$$
\hat{\omega}(U) \subset f^{\omega}(U) .
$$

Proof. Let $g \equiv \bigcup_{k \geq n} f^{k}$ and use Lemmas 2.4, 3.5 and 9.14.

Remark 9.16. It is not necessarily true that $\hat{\omega}(U)=f^{\omega}(U)$, not even for maps, as the following example shows. Let $X \equiv[0,1]$ and

$$
f \equiv\left\{(x, y) \in X \times X: y=x^{2}\right\}
$$

It is easy to check that

$$
f^{\omega}=\{(x, y) \in X \times X: x=0 \text { or } y=1\} .
$$

Clearly $\hat{\omega}(\{1\})=\{1\}$, but $f^{\omega}(\{1\})=X$. 
The following theorem characterizes the nonwandering set of a relation as the fixed point set of the relation $f^{\omega}$.

Theorem 9.17. If $f$ is a closed relation on a compact Hausdorff space $X$, then

$$
\mathrm{N}_{\omega}(f)=\mathrm{F}\left(f^{\omega}\right) .
$$

Proof. Let $x \notin \mathrm{F}\left(f^{\omega}\right)$. This means $x \notin f^{\omega}(x)$. By Lemma 4.5 there exists $V \in \overline{\mathfrak{N}}(x)$ such that $x \notin f^{\omega}(V)$, which by Lemma 9.15 means $x \notin \hat{\omega}(V)$, but then $x \notin \mathrm{N}_{\omega}(f)$. This implies that

$$
\mathrm{N}_{\omega}(f) \subset \mathrm{F}\left(f^{\omega}\right)
$$

Now let $x \notin \mathrm{N}_{\omega}(f)$. This means there exists a $W$ neighborhood of $x$, such that $x \notin \hat{\omega}(W) . \hat{\omega}(W)$ is a closed set by its definition, $x$ is closed, so there exists $V \in \overline{\mathfrak{N}}(x)$ such that $V \subset W$ and $V \cap \hat{\omega}(W)=\emptyset$. Clearly $\hat{\omega}(V) \subset \hat{\omega}(W)$, so $V \cap \hat{\omega}(V)=\emptyset$. Suppose now that $x \in \mathrm{F}\left(f^{\omega}\right)$. This means that for every $n \geq 0$

$$
(x, x) \in \overline{\bigcup_{k \geq n} f^{k}},
$$

which means that for every $n \geq 0$ and for every $U$ neighborhood of $x$

$$
(U \times U) \cap\left(\bigcup_{k \geq n} f^{k}\right) \neq \emptyset
$$

which is equivalent to

$$
\bigcup_{k \geq n}\left((U \times U) \cap f^{k}\right) \neq \emptyset .
$$

This implies that for every $n \geq 0$ and for every $U$ neighborhood of $x$ there exists $k \geq n$ such that

$$
f^{k}(U) \cap U \neq \emptyset
$$

But this means that the above statement is true for $V$, i.e. for every $n \geq 0$ there exists $k \geq n$ such that

$$
f^{k}(V) \cap V \neq \emptyset
$$

Then for every $n \geq 0$

$$
\bigcup_{k \geq n}\left(f^{k}(V) \cap V\right) \neq \emptyset
$$

which is equivalent to

$$
\left(\bigcup_{k \geq n} f^{k}(V)\right) \cap V \neq \emptyset .
$$

But then for every $n \geq 0$

$$
\left(\overline{\bigcup_{k \geq n} f^{k}(V)}\right) \cap V \neq \emptyset
$$

which implies

and we conclude

$$
\bigcap_{n \geq 0}\left(\overline{\bigcup_{k \geq n} f^{k}(V)} \cap V\right) \neq \emptyset
$$

$$
V \cap \hat{\omega}(V)=V \cap\left(\bigcap_{n \geq 0} \overline{\bigcup_{k \geq n} f^{k}(V)}\right) \neq \emptyset
$$

which is a contradiction, so $x \notin \mathrm{F}\left(f^{\omega}\right)$ and this implies that

$$
\mathrm{F}\left(f^{\omega}\right) \subset \mathrm{N}_{\omega}(f)
$$


and completes the proof.

Corollary 9.18. If $f$ is a closed relation on a compact Hausdorff space $X$, then $\mathrm{N}_{\omega}(f)$ is closed.

Proof. This is an immediate consequence of Theorem 7.2, Lemma 9.4 and Theorem 9.17 .

The next part will concern the chain recurrent set. In order to give motivation to this part, first we need the notion of a chain or pseudo-orbit for a map.

Definition 9.19. If $f$ is a map on a metric space $X$, then an $\varepsilon$-chain (or $\varepsilon$-pseudoorbit) for $f$ is a sequence of points $p_{i} \in X, i=0,1, \ldots n$ satisfying $d\left(p_{i+1}, f\left(p_{i}\right)\right) \leq \varepsilon$.

We have the following well-known definition for the chain recurrent set of a map on a metric space $X$.

Definition 9.20. If $f$ is a map on a metric space $X$, then $x \in X$ is called chain recurrent if for every $\varepsilon>0$ there exists an $\varepsilon$-chain $\left\{p_{i}: i=0,1, \ldots n\right\}$ such that $p_{0}=p_{n}=x$. The set of chain recurrent points is usually denoted by $\mathrm{R}(f)$.

Now it is easy to see, that if $f$ is a map on a metric space $X$, then an $\varepsilon$-chain for $f$ is an orbit for the relation

$$
f_{\varepsilon} \equiv\{(x, y): d(y, f(x)) \leq \varepsilon\} .
$$

This means we have the following theorem, which is an immediate consequence of the above definitions.

Theorem 9.21. If $f$ is a map on a metric space $X$, then

$$
\mathrm{R}(f)=\bigcap_{\varepsilon>0} \mathrm{P}\left(f_{\varepsilon}\right)
$$

The relation $f_{\varepsilon}$ is clearly a neighborhood of the map, and hence the relation $f$. A natural generalization of this definition is then the following.

Definition 9.22. If $f$ is a closed relation on a compact Hausdorff space $X$, then $x \in X$ is called chain recurrent for $f$ if for every closed neighborhood $\phi$ of $f, x$ is periodic for $\phi$. The set of chain recurrent points will be denoted by

$$
\mathrm{R}(f)=\bigcap_{\phi \in \overline{\mathfrak{N}}(f)} \mathrm{P}(\phi) .
$$

The following theorem characterizes the chain recurrent set of a relation as the fixed point set of the relation $f^{\Omega}$.

Theorem 9.23. If $f$ is a closed relation on a compact Hausdorff space $X$, then

$$
\mathrm{R}(f)=\mathrm{F}\left(f^{\Omega}\right) .
$$

Proof. Lemma 9.6 and Theorem 9.8 imply that

$$
\mathrm{R}(f)=\bigcap_{\phi \in \overline{\mathfrak{N}}(f)} \mathrm{F}\left(\phi^{\infty}\right)=\mathrm{F}\left(\bigcap_{\phi \in \overline{\mathfrak{N}}(f)} \phi^{\infty}\right)=\mathrm{F}\left(f^{\Omega}\right)
$$

and the proof is complete.

Corollary 9.24. If $f$ is a closed relation on a compact Hausdorff space $X$, then $\mathrm{R}(f)$ is closed. 
Proof. This is an immediate consequence of Theorem 8.3, Lemma 9.4 and Theorem 9.23 . Let

The last part of this section is a construction of an equivalence relation on $\mathrm{R}(f)$.

$$
\mathcal{E}(f) \equiv f^{\Omega} \cap\left(f^{\Omega}\right)^{*} .
$$

We claim that this is an equivalence relation on $\mathrm{R}(f)$.

Lemma 9.25. If $f$ is a closed relation on a compact Hausdorff space $X$, then the following properties hold.

(a) $\mathcal{E}(f)=\mathcal{E}\left(f^{*}\right)$.

(b) $\mathcal{E}(f)$ is an equivalence relation on $\mathrm{R}(f)$.

Proof. Property (a) is an immediate consequence of the construction and Theorem 8.2 .

Clearly $\mathcal{E}(f) \subset \mathrm{R}(f) \times \mathrm{R}(f)$ : if $(x, y) \in \mathcal{E}(f)$, then $(x, y) \in f^{\Omega},(x, y) \in\left(f^{*}\right)^{\Omega}=$ $\left(f^{\Omega}\right)^{*}$, so $(y, x) \in f^{\Omega}$, but then by Theorem $8.5(x, x) \in f^{\Omega}$ and $(y, y) \in f^{\Omega}$, which means $(x, y) \in \mathrm{R}(f) \times \mathrm{R}(f)$. To prove that $\mathcal{E}(f)$ is an equivalence relation on $\mathrm{R}(f)$, we show that $\mathcal{E}(f)$ is reflexive, symmetric and transitive. Reflexivity: if $x \in \mathrm{R}(f)$, then $(x, x) \in f^{\Omega}$, so $(x, x) \in\left(f^{\Omega}\right)^{*}=\left(f^{*}\right)^{\Omega}$, but then $(x, x) \in f^{\Omega} \cap\left(f^{\Omega}\right)^{*}=\mathcal{E}(f)$. Symmetry: this is trivial by the construction of $\mathcal{E}(f)$, using again Theorem 8.2. Transitivity: if $(x, y) \in \mathcal{E}(f)$ and $(y, z) \in \mathcal{E}(f)$, then again by the construction and by Theorem $8.5(x, z) \in f^{\Omega}$ and $(z, x) \in f^{\Omega}$, which implies that $(x, z) \in \mathcal{E}(f)$.

Definition 9.26. The equivalence classes on $\mathrm{R}(f)$ defined by this equivalence relation are called chain components.

We close this section with the following easy lemma.

Lemma 9.27. If $f$ is a closed relation on a compact Hausdorff space $X$ and $(x, y) \in$ $\mathcal{E}(f)$, then $f^{\Omega}(x)=f^{\Omega}(y)$.

Proof. Let $z \in f^{\Omega}(x)$. This means $(x, z) \in f^{\Omega}$, and because $(x, y) \in \mathcal{E}(f)$ we know $(y, x) \in f^{\Omega}$. By Theorem 8.5 this means that $(y, z) \in f^{\Omega}$, so $z \in f^{\Omega}(y)$, which proves $f^{\Omega}(x) \subset f^{\Omega}(y)$, but then by the symmetry of $\mathcal{E}(f)$ clearly $f^{\Omega}(y) \subset f^{\Omega}(x)$ and we proved the claim.

\section{Attractors AND REPELlERS}

The concept of attraction is widely used in dynamical systems theory. First we will define the notion of attractor for closed relations, then we establish the corresponding theory about repellers and connecting orbits. In the subsequent sections we prove that this notion is really a generalization of the well-known definitions of attractor for maps.

Definition 10.1. If $f$ is a closed relation on a compact Hausdorff space $X$, then $A \subset X$ is called an attractor for $f$ if there exists $G \in \overline{\mathfrak{N}}(A)$ such that

$$
f^{\Omega}(G)=A \text {. }
$$

The set of attractors for $f$ will be denoted by $\mathfrak{A}(f)$. The basin of the attractor $A$ is the set

$$
\mathrm{B}(A) \equiv\left(f^{\Omega}\right)^{-1}(A) .
$$

The following lemma gives some fundamental properties of attractors. 
Lemma 10.2. If $A$ is an attractor for a closed relation $f$ on a compact Hausdorff space $X$, then the following properties hold.
(a) $A$ is closed.
(b) $f^{\Omega}(A)=f(A)=A$.
(c) $S \subset \mathrm{B}(A)$ if and only if $f^{\Omega}(S) \subset A$.
(d) $A \subset \mathrm{B}(A)$.
(e) $f^{\Omega}(\mathrm{B}(A))=A$.

Proof. By definition, there exists $G \in \overline{\mathfrak{N}}(A)$ such that $f^{\Omega}(G)=A$. Since $G$ is closed, Corollary 8.4 implies that $A$ is closed, and property (a) is established. Property (b) is an immediate consequence of Theorem 8.5, while property (c) follows immediately from Lemma 3.8. Property (d) is implied by properties (b) and (c). Properties (b), (c) and (d) imply that

$$
A=f^{\Omega}(A) \subset f^{\Omega}(\mathrm{B}(A)) \subset A
$$

and we verified property (e) and the proof is complete.

Our next notion is the dual notion to attractor: the repeller.

Definition 10.3. If $A$ is an attractor for a closed relation $f$ on a compact Hausdorff space $X$, then the dual repeller of $A$ is the set

$$
A^{*} \equiv \mathrm{B}(A)^{c} .
$$

It is natural to think that $A^{*}$ is an attractor for the "backward" relation, i.e. for the transpose. It will turn out that this is indeed the case. First we prove the following lemma.

Lemma 10.4. If $A$ is an attractor for a closed relation $f$ on a compact Hausdorff space $X$, then

$$
A^{*}=\left(f^{*}\right)^{\Omega}\left(A^{c}\right) .
$$

Proof. The definitions, Lemma 3.12 and Theorem 8.2 imply that

$$
A^{*}=\mathrm{B}(A)^{c}=\left(\left(f^{\Omega}\right)^{-1}(A)\right)^{c}=\left(f^{\Omega}\right)^{*}\left(A^{c}\right)=\left(f^{*}\right)^{\Omega}\left(A^{c}\right),
$$

and the proof is complete.

Theorem 10.5. If $A$ is an attractor for a closed relation $f$ on a compact Hausdorff space $X$, then $A^{*}$ is an attractor for $f^{*}$.

Proof. First note that Lemma 4.2, Theorem 8.5 and Lemma 10.4 imply that

$$
\left(f^{*}\right)^{\Omega}\left(A^{*}\right)=A^{*} .
$$

Next note that, by definition, there exists $G \in \overline{\mathfrak{N}}(A)$ such that $f^{\Omega}(G)=A$. Lemma 10.2 implies that $G \subset \mathrm{B}(A)$. By the definition of neighborhood there exists an open set $U$ such that

which implies that

$$
A \subset U \subset G \subset \mathrm{B}(A),
$$

$$
A^{*}=\mathrm{B}(A)^{c} \subset G^{c} \subset U^{c} \subset A^{c} .
$$

Since $G^{c}$ is open and $U^{c}$ is closed, $U^{c} \in \overline{\mathfrak{N}}\left(A^{*}\right)$. Equation (7), Lemma 3.1 and Lemma 10.4 imply that

$$
A^{*}=\left(f^{*}\right)^{\Omega}\left(A^{*}\right) \subset\left(f^{*}\right)^{\Omega}\left(U^{c}\right) \subset\left(f^{*}\right)^{\Omega}\left(A^{c}\right)=A^{*} .
$$

Therefore, $A^{*}=\left(f^{*}\right)^{\Omega}\left(U^{c}\right)$, and the proof is complete. 
Because $A^{*}$ is an attractor for $f^{*}$, we can create the dual repeller to it. The following theorem justifies the name "dual repeller" in the definition.

Theorem 10.6. If $A$ is an attractor for a closed relation $f$ on a compact Hausdorff space $X$, then

$$
\left(A^{*}\right)^{*}=A,
$$

where the second ${ }^{*}$ operation corresponds to $f^{*}$.

Proof. Using the definition of basin and dual repeller and Lemma 3.12, Theorem 8.2, Lemma 3.10 and Lemma 10.2 we deduce

$$
\begin{aligned}
\left(A^{*}\right)^{*} & =\mathrm{B}\left(A^{*}\right)^{c}=\left(\left(f^{*}\right)^{\Omega}\right)^{-1}\left(A^{*}\right)^{c}=\left(\left(f^{*}\right)^{\Omega}\right)^{*}\left(\left(A^{*}\right)^{c}\right)= \\
& =\left(f^{\Omega}\right)\left(\left(A^{*}\right)^{c}\right)=f^{\Omega}(\mathrm{B}(A))=A
\end{aligned}
$$

and the proof is complete.

Lemma 10.7. If $A$ is an attractor for a closed relation $f$ on a compact Hausdorff space $X, x \in A$ and $y \in A^{*}$, then $(x, y) \notin \mathcal{E}(f)$.

Proof. By Lemma $10.2 f^{\Omega}(x) \subset f^{\Omega}(A)=A$ and $f^{\Omega}(y) \not \subset A$, this implies $f^{\Omega}(x) \neq$ $f^{\Omega}(y)$ and Lemma 9.27 implies that $(x, y) \notin \mathcal{E}(f)$ and the proof is complete.

The following lemma investigates the basin.

Lemma 10.8. If $A$ is an attractor for a closed relation $f$ on a compact Hausdorff space $X$, then the following properties hold.

(a) $\mathrm{B}(A)$ is open.

(b) $f^{-1}(\mathrm{~B}(A))=\mathrm{B}(A)$.

Proof. Property (a) is an immediate consequence of the definitions, Theorem 10.5 and Lemma 10.2. By Lemmas 3.12 and 10.2 and Theorem 10.5

$$
f^{-1}(\mathrm{~B}(A))^{c}=f^{*}\left(\mathrm{~B}(A)^{c}\right)=f^{*}\left(A^{*}\right)=A^{*}=\mathrm{B}(A)^{c}
$$

and we established property (b).

We introduced two fundamental objects, the attractor and the corresponding dual repeller. We give the following name for the remaining part of the space.

Definition 10.9. If $A$ is an attractor for a closed relation $f$ on a compact Hausdorff space $X$, then the set of connecting orbits associated with $A$ is given by

$$
\mathrm{C}(A) \equiv\left(A \cup A^{*}\right)^{c} \text {. }
$$

Lemma 10.10. If $A$ is an attractor for a closed relation $f$ on a compact Hausdorff space $X$, then $X$ breaks into the disjoint union of $A, A^{*}$ and $\mathrm{C}(A)$.

Proof. By Lemma $10.2 A \subset \mathrm{B}(A)=\left(A^{*}\right)^{c}$, so we conclude that $A \cap A^{*}=\emptyset$, and the definition of $\mathrm{C}(A)$ completes the proof.

We close this section with the following lemma, which will play a key role in the proof of the Conley decomposition theorem. The lemma gives a method for creating an attractor for a relation.

Lemma 10.11. If $f$ is a closed relation on a compact Hausdorff space $X, g$ is a closed neighborhood of $f$ and $K$ is a closed subset of $X$, then $f^{\Omega}\left(g^{\Omega}(K)\right)$ is an attractor for $f$. 
Proof. Let

$$
G \equiv g^{\Omega}(K) \quad \text { and } \quad A \equiv f^{\Omega}(G) .
$$

Corollary 8.4 implies that $G$ is closed, while Theorem 8.5 implies that $g^{\Omega}(G)=G$. Since Lemma 8.6 implies that $g^{\Omega} \in \overline{\mathfrak{N}}\left(f^{\Omega}\right)$, Lemma 4.6 implies that

$$
G=g^{\Omega}(G) \in \overline{\mathfrak{N}}\left(f^{\Omega}(G)\right)=\overline{\mathfrak{N}}(A),
$$

which implies that $A$ is an attractor and completes the proof.

\section{Aтtractor BLOCKS}

In order to establish that the notion of attractor defined in the previous section gives the same objects for maps as the already well-known definitions, we will have to introduce the following important construction.

Definition 11.1. If $f$ is a closed relation on a compact Hausdorff space $X$, then $B \subset X$ is called an attractor block for $f$ if

$$
f(\bar{B}) \subset B^{\circ} .
$$

A basic property of an attractor is that it can be surrounded by an attractor block; conversely, if we have an attractor block, (i.e. a set whose image lies strictly inside itself) then inside the attractor block we have an attractor. Our aim in this section is to prove this connection between attractors and attractor blocks. Note that in the definition of the attractor block we did not use any of the constructions given before: it is strictly a statement about the image of a set under the relation.

First we prove the following lemma.

Lemma 11.2. If $A$ is an attractor for a closed relation $f$ on a compact Hausdorff space $X$, then for any $V \in \mathfrak{N}(A)$ there exists $g \in \overline{\mathfrak{N}}(f)$ such that $g^{\Omega}(A) \subset V^{o}$.

Proof. By Lemma 4.4 there exists $\phi \in \mathfrak{N}^{o}(f)$ such that $\phi(A) \subset V^{o}$. Theorem 8.8 implies there exists $g \in \overline{\mathfrak{N}}(f)$ such that $g^{\Omega} \subset \phi$. Then $g^{\Omega}(A) \subset \phi(A) \subset V^{o}$ and the proof is complete.

The following two theorems give the above mentioned connection between attractors and attractor blocks: every attractor block has an attractor in its interior and every attractor can be surrounded by an attractor block.

Theorem 11.3. If $B$ is an attractor block for a closed relation $f$ on a compact Hausdorff space $X$, then $f^{\Omega}(B) \subset B^{o}$ and $f^{\Omega}(B)$ is an attractor for $f$.

Proof. First we prove that $f^{\Omega}\left(B^{o}\right)=f^{\Omega}(B)=f^{\Omega}(\bar{B})$. By the assumption $f(\bar{B}) \subset$ $B^{\circ}$ and by Theorem 8.5

$$
f^{\Omega}(\bar{B})=f^{\Omega}(f(\bar{B})) \subset f^{\Omega}\left(B^{o}\right),
$$

by Lemma $3.1 f^{\Omega}\left(B^{o}\right) \subset f^{\Omega}(B) \subset f^{\Omega}(\bar{B})$ and we conclude that $f^{\Omega}\left(B^{o}\right)=f^{\Omega}(B)=$ $f^{\Omega}(\bar{B})$.

Next we prove that $f^{\Omega}(\bar{B}) \subset \bar{B}$. By the definition of $f^{\Omega}$ and Lemma 3.5

$$
f^{\Omega}(\bar{B})=\left(\bigcap_{\phi \in \overline{\mathfrak{N}}(f)} \phi^{\infty}\right)(\bar{B})=\bigcap_{\phi \in \overline{\mathfrak{N}}(f)} \phi^{\infty}(\bar{B})
$$


This means we have to prove that there exists $\phi \in \overline{\mathfrak{N}}(f)$ such that $\phi^{\infty}(\bar{B}) \subset \bar{B}$. In order to prove this statement, we first show that there exists $\phi \in \overline{\mathfrak{N}}(f)$ such that $\phi(\bar{B}) \subset \bar{B}$. By assumption $f(\bar{B}) \subset B^{o}$, by Lemma 3.3 this means that

$$
\left(\bar{B} \times\left(B^{o}\right)^{c}\right) \cap f=\emptyset \text {. }
$$

$\bar{B},\left(B^{o}\right)^{c}$ and $f$ is closed, so by the normality of $X \times X$ there exists $\phi \in \overline{\mathfrak{N}}(f)$ such that

$$
\left(\bar{B} \times\left(B^{o}\right)^{c}\right) \cap \phi=\emptyset,
$$

which means $\phi(\bar{B}) \subset B^{o} \subset \bar{B}$. Now by an induction argument we get easily that $\phi^{k}(\bar{B})=\phi\left(\phi^{k-1}(\bar{B})\right) \subset \phi(\bar{B}) \subset \bar{B}$ for any $k>0$, this means $\bigcup_{k \geq n} \phi^{k}(\bar{B}) \subset \bar{B}$ for any $n \geq 0$ and then by Lemma 3.5

$$
\phi^{\infty}(\bar{B})=\bigcap_{n \geq 0} \bigcup_{k \geq n} \phi^{k}(\bar{B}) \subset \bar{B} .
$$

We proved that there exists $\phi \in \overline{\mathfrak{N}}(f)$ such that $\phi^{\infty}(\bar{B}) \subset \bar{B}$, and then we established that $f^{\Omega}(\bar{B}) \subset \bar{B}$. Now with the aid of Theorem 8.5 and the above

$$
f^{\Omega}(B)=f^{\Omega}(\bar{B})=f\left(f^{\Omega}(\bar{B})\right) \subset f(\bar{B}) \subset B^{o} \subset \bar{B}
$$

which means that $\bar{B}$ is a neighborhood of $f^{\Omega}(\bar{B})$ and by the definition of attractor this means that $f^{\Omega}(B)$ is an attractor for $f$ and the proof is complete.

Theorem 11.4. If $A$ is an attractor for a closed relation $f$ on a compact Hausdorff space $X$ and $V$ is a neighborhood of $A$, then there exists a closed attractor block $B$ for $f$ such that $B \subset V$ and $f^{\Omega}(B)=A$.

Proof. By Lemma $10.8 \mathrm{~B}(A)$ is open and by Lemma $10.2 A \subset \mathrm{B}(A)$. By assumption $A \subset V^{o}$, this means that $W \equiv \mathrm{B}(A) \cap V^{o} \in \mathfrak{N}^{o}(A)$. Now by Lemma 11.2 there exists $g \in \overline{\mathfrak{N}}(f)$ such that

$$
g^{\Omega}(A) \subset W \subset \mathrm{B}(A) .
$$

Let $B \equiv g^{\Omega}(A)$. By Lemma 10.2, Theorem 8.3 and Lemma $4.10 B$ is closed. By Lemma 10.2

$$
f^{\Omega}(B)=f^{\Omega}\left(g^{\Omega}(A)\right) \subset f^{\Omega}(\mathrm{B}(A))=A .
$$

On the other hand, $A=f^{\Omega}(A) \subset g^{\Omega}(A)=B$, then by Theorem 8.5

$$
A=f^{\Omega}(A)=f^{\Omega}\left(f^{\Omega}(A)\right) \subset f^{\Omega}(B),
$$

which means $A=f^{\Omega}(B)$. In order to finish the proof, we have to prove only that $B$ is an attractor block, i.e. $f(\bar{B})=f(B) \subset B^{o} . g$ is a closed neighborhood of $f$, this implies that there exists an open $\phi$ such that $f \subset \phi \subset g$. Then

$$
f(B) \subset \phi(B) \subset g(B)=g\left(g^{\Omega}(A)\right)=g^{\Omega}(A)=B
$$

and by Lemma $4.3 \phi(B)$ is open, which means $B$ is really an attractor block for $f$ and the proof is complete.

We close this section with the following theorem, which basically states that attractors are "robust for small perturbations".

Theorem 11.5. [12] If $A$ is an attractor for a closed relation $f$ on a compact Hausdorff space $X$, then for any $V \in \mathfrak{N}(A)$ there exists $g \in \overline{\mathfrak{N}}(f)$ such that $g$ has an attractor $A^{\prime}$ in $V$ and $A \subset A^{\prime}$. 
Proof. Lemma 11.2 implies there exists $h \in \overline{\mathfrak{N}}(f)$ such that $h^{\Omega}(A) \subset V^{o}$. Let $B \equiv h^{\Omega}(A)$. By the normality of $X$ there exists $g \in \overline{\mathfrak{N}}(f)$ such that $h \in \overline{\mathfrak{N}}(g)$, i.e. $g \subset \xi \subset h$ where $\xi$ is open. By Lemma 10.2, Theorem 8.3 and Lemma $4.10 B$ is closed. Then by Theorem 8.5

$$
g(B)=g\left(h^{\Omega}(A)\right) \subset \xi\left(h^{\Omega}(A)\right) \subset h\left(h^{\Omega}(A)\right)=h^{\Omega}(A)=B,
$$

and by Lemma $4.3 \xi(B)$ is open, which means $B$ is an attractor block for $g$ and Theorem 11.3 implies the claim.

\section{Comparison}

In [8], McGehee defined attractors for closed relations on compact Hausdorff spaces in a different way. He proved that that notion is a natural generalization of attractors for maps and as such, in the special case of maps the definition gives us the already known ones. In this section we give this alternative definition for attractors, then we show that the two definitions are equivalent.

First we will define the omega limit set of a set. Recall Definition 5.8. Now if $f$ is a closed relation on a compact Hausdorff space $X$ and $S \subset X$, then let

$\mathfrak{K}(S) \equiv\left\{K: K\right.$ is a closed confining set satisfying $f^{n}(S) \subset K$ for some $\left.n \geq 0\right\}$.

Definition 12.1. [8] The omega limit set of a set $S$ under the relation $f$ is the set

$$
\omega(S) \equiv \bigcap \mathfrak{K}(S) .
$$

Now we are ready for the alternative definition of an attractor for a closed relation $f$ on a compact Hausdorff space $X$. Compare this definition to Definition 10.1.

Definition 12.2. [8] If $f$ is a closed relation on a compact Hausdorff space $X$, then $A \subset X$ is called an attractor for $f$ if there exists $G \in \overline{\mathfrak{N}}(A)$ such that

$$
\omega(G)=A \text {. }
$$

Remark 12.3. It is not necessarily true that for $G \subset X, f^{\Omega}(G)=\omega(G)$. Consider the example of Remark 9.16. It is easy to check that

$$
f^{\Omega}=\{(x, y) \in X \times X: x=0 \text { or } y=1\} .
$$

Clearly $\omega(\{1\})=\{1\}$, but $f^{\Omega}(\{1\})=X$.

We will show now that the two definitions are equivalent, i.e. if $A$ is an attractor according to one of the definitions, then it is an attractor according to the other definition as well.

Theorem 12.4. The two definitions of the attractor for a closed relation are equivalent.

Proof. Let $f$ be a closed relation on a compact Hausdorff space $X, A$ be an attractor according to Definition 12.2 and $V \in \mathfrak{N}(A)$. By Theorem 7.3 in [8], there exists a closed attractor block $B$ for $f$ such that $B \subset V$ and $\omega(B)=A$. We will show that $\omega(B)$ is a closed invariant set in $B$, which is not contained in any other closed invariant set in $B$. By Lemma 7.1 in $[8] \omega(B)=A$ is a closed invariant set. Suppose now that $C \subset B, f(C)=C, C$ is closed and $\omega(B)=A \subset C$. By Theorem 5.10 in [8], if $S \subset S^{\prime}$, then $\omega(S) \subset \omega\left(S^{\prime}\right)$. By Theorem 5.12 in [8], if $S$ is a closed invariant set, then $\omega(S)=S$. $C$ is a closed invariant set, this means

$$
C=\omega(C) \subset \omega(B) \subset C,
$$


but this implies $C=\omega(B)=A$, so we conclude that $A$ is a closed invariant set in the attractor block $B$, which is not contained in any other closed invariant set in $B$. Consider now $f^{\Omega}(B)$. It is a closed, invariant set in $B$ according to Theorem 11.3. Suppose $f^{\Omega}(B) \subset C$, where $C$ is a closed invariant set in $B$. Then $f^{\Omega}(B) \subset C \subset B$, and by Theorem 8.5

$$
f^{\Omega}(B)=f^{\Omega}\left(f^{\Omega}(B)\right) \subset f^{\Omega}(C) \subset f^{\Omega}(B),
$$

which means $f^{\Omega}(C)=f^{\Omega}(B)$, and because $C$ is invariant, $C \subset f^{\Omega}(C)=f^{\Omega}(B)$ and then $C=f^{\Omega}(B)$, so $f^{\Omega}(B)$ is a closed invariant set in $B$, which is not contained in any other closed invariant set in $B$. If $S$ and $T$ are closed invariant sets, then $S \cup T$ is closed and invariant. This implies that $f^{\Omega}(B)=\omega(B)=A$. B is a closed attractor block for $f$, and then by Theorem $11.3 f^{\Omega}(B)=A$ is an attractor for $f$ according to Definition 10.1. The proof of the opposite direction is identical.

\section{Decomposition}

In this section we prove the main result: the generalization of Conley's decomposition theorem for relations, which states that the chain recurrent set is determined by the attractors and dual repellers. Recall that the set of attractors for a closed relation $f$ is denoted by $\mathfrak{A}(f)$.

Theorem 13.1. If $f$ is a closed relation on a compact Hausdorff space $X$, then

$$
\mathrm{R}(f)^{c}=\bigcup_{A \in \mathfrak{A}(f)} \mathrm{C}(A) .
$$

Proof. Let $x \in \mathrm{C}(A)$ for some attractor $A$. Since $x \in \mathrm{B}(A)$, it follows that $f^{\Omega}(x) \subset$ $A$, which, since $x \notin A$, implies that $x \notin f^{\Omega}(x)$. Therefore, $x \notin \mathrm{R}(f)$, which establishes that

$$
\bigcup_{A \in \mathfrak{A}(f)} \mathrm{C}(A) \subset \mathrm{R}(f)^{c} .
$$

Now suppose that $x \in \mathrm{R}(f)^{c}$, this is equivalent to $(x, x) \notin f^{\Omega}$. Now by Lemma 8.7 there exists $g \in \overline{\mathfrak{N}}(f)$, such that $(x, x) \notin g^{\Omega}$. This means $x \notin g^{\Omega}(x) \equiv U$. Now by Lemma $10.11 A=f^{\Omega}\left(g^{\Omega}(x)\right)=f^{\Omega}(U)$ is an attractor for $f$. Also, by Lemma $8.6 g^{\Omega} \in \overline{\mathfrak{N}}\left(f^{\Omega}\right)$, and by Lemma 4.6 and Theorem 8.5 it follows that

$$
U=g^{\Omega}(U) \in \overline{\mathfrak{N}}\left(f^{\Omega}(U)\right)=\overline{\mathfrak{N}}(A)
$$

and then $x \notin U$ implies that $x \notin A$. On the other hand,

$$
f^{\Omega}(x) \subset g^{\Omega}(x)=U,
$$

which implies, together with Theorem 8.5 that

$$
f^{\Omega}(x)=f^{\Omega}\left(f^{\Omega}(x)\right) \subset f^{\Omega}(U)=A,
$$

which implies $x \in\left(f^{\Omega}\right)^{-1}(A)$, i.e., $x \in \mathrm{B}(A)$, and then $x \notin A^{*}$. This together with $x \notin A$ implies that

$$
\mathrm{R}(f)^{c} \subset \bigcup_{A \in \mathfrak{A}(f)} \mathrm{C}(A)
$$

and completes the proof. 


\section{Simple EXAMPLES}

We give some illustrating examples in this section. The topology is the standard topology of $\mathbb{R}^{1}$ restricted to the given closed intervals.

Example 14.1. Let $X \equiv[0,1]$ and $f \equiv \iota$. By the definitions it is easy to check that

$$
f^{\infty}=f^{\omega}=\iota
$$

and

$$
f^{\Omega}=X \times X .
$$

We can see from here that $X$ is an attractor with dual repeller $\emptyset$ and $\emptyset$ is an attractor with dual repeller $X . \mathrm{C}(X)=\emptyset, \mathrm{C}(\emptyset)=\emptyset$ and we see that

$$
\mathrm{F}\left(f^{\Omega}\right)=\mathrm{R}(f)=\left(\bigcup_{A \in \mathfrak{A}(f)} \mathrm{C}(A)\right)^{c}=X .
$$

Also, $\mathcal{E}(f)=X \times X$ and we have one chain component: $X$.

Example 14.2. Let $X \equiv[0,1]$ and

$$
f \equiv\left\{(x, y) \in X \times X: y=x^{2}\right\} .
$$

By the definitions it is easy to check that

$$
f^{\infty}=\{(0,0)\} \cup\{(1,1)\}
$$

and

$$
f^{\omega}=f^{\Omega}=\{(x, y) \in X \times X: x=1 \text { or } y=0\} .
$$

We can see from here that $\{0\}$ is an attractor with dual repeller $\{1\}$. Also, $X$ is an attractor with dual repeller $\emptyset$ and $\emptyset$ is an attractor with dual repeller $X$. $\mathrm{C}(\{0\})=(0,1), \mathrm{C}(X)=\emptyset, \mathrm{C}(\emptyset)=\emptyset$ and we see that

$$
\mathrm{F}\left(f^{\Omega}\right)=\mathrm{R}(f)=\left(\bigcup_{A \in \mathfrak{A}(f)} \mathrm{C}(A)\right)^{c}=\{0,1\} .
$$

Also, $\mathcal{E}(f)=\{(0,0),(1,1)\}$ and we have two chain components: $\{0\}$ and $\{1\}$.

Example 14.3. Let $X \equiv[0,1]$ and

$$
f \equiv\{(0,1)\} \cup\{(x, y) \in X \times X: x=2 y\} .
$$

Also, let $S \equiv\left\{1 / 2^{n}: n=0,1,2, \ldots\right\} \cup\{0\} \subset X$. By the definitions it is easy to check that

$$
\begin{gathered}
f^{\infty}=\{(0, x): x \in S\}, \\
f^{\omega}=\{(0, x): x \in S\} \cup\{(x, 0): x \in X\}
\end{gathered}
$$

and

$$
f^{\Omega}=\{(x, y) \in X \times X: x \in X, y \in S\} .
$$

We can see from here that $S \subset X$ is an attractor with dual repeller $\emptyset$. Also, $\emptyset$ is an attractor with dual repeller $X . \mathrm{C}(S)=S^{c}, \mathrm{C}(\emptyset)=\emptyset$ and we see that

$$
\mathrm{F}\left(f^{\Omega}\right)=\mathrm{R}(f)=\left(\bigcup_{A \in \mathfrak{A}(f)} \mathrm{C}(A)\right)^{c}=S .
$$

Also, $\mathcal{E}(f)=S \times S$ and we have one chain component: $S$. 
The next two examples are familiar from the classical theory of maps. The second example is the general case, but the first helps to see the structure of these relations.

Example 14.4. Let $X \equiv[-1,1], 1<\alpha$ and

$$
f \equiv\{(x, y) \in X \times X: y=\alpha x\} .
$$

By the definitions it is easy to check that

$$
f^{\infty}=\{(0,0)\}
$$

and

$$
f^{\omega}=f^{\Omega}=\{(0, x): x \in X\} .
$$

We can see from here that $X$ is an attractor with dual repeller $\emptyset$. Also, $\emptyset$ is an attractor with dual repeller $\{0\} . \mathrm{C}(X)=\emptyset, \mathrm{C}(\emptyset)=\{0\}^{c}$ and we see that

$$
\mathrm{F}\left(f^{\Omega}\right)=\mathrm{R}(f)=\left(\bigcup_{A \in \mathfrak{A}(f)} \mathrm{C}(A)\right)^{c}=\{0\} .
$$

Also, $\mathcal{E}(f)=\{(0,0)\}$ and we have one chain component: $\{0\}$.

Example 14.5. Let $X \equiv[-1,1] \times[-1,1], 0<\beta<1<\alpha$ and

$$
f \equiv\{(x, y) \in X \times X: y=A x\}
$$

where

$$
A=\left[\begin{array}{ll}
\alpha & 0 \\
0 & \beta
\end{array}\right] \text {. }
$$

By the definitions it is easy to check that

$$
f^{\infty}=\{((0,0),(0,0))\}
$$

and

$$
f^{\omega}=f^{\Omega}=\{((0, x),(y, 0)): x, y \in[-1,1]\} .
$$

We can see from here that $H \equiv f^{\Omega}(X)=\{(x, 0): x \in[-1,1]\}$ (the horizontal axis) is an attractor with dual repeller $\emptyset$. Also, $\emptyset$ is an attractor with dual repeller $V \equiv$ $\left(\left(f^{\Omega}\right)^{-1}(\emptyset)\right)^{c}=\{(0, x): x \in[-1,1]\}$ (the vertical axis). $\mathrm{C}(H)=H^{c}, \mathrm{C}(\emptyset)=V^{c}$ and we see that

$$
\mathrm{F}\left(f^{\Omega}\right)=\mathrm{R}(f)=\left(\bigcup_{A \in \mathfrak{A}(f)} \mathrm{C}(A)\right)^{c}=\{(0,0)\} .
$$

Also, $\mathcal{E}(f)=\{((0,0),(0,0))\}$ and we have one chain component: $\{(0,0)\}$.

Barnsley has studied fractals generated by sets of contraction mappings (called Iterated Function Systems or IFS), i.e. relations in [2]. It can be shown that his definition of attractor for IFS is identical to the one given here (for a proof, see [8]). The next example possesses the standard middle-third Cantor set as an attractor.

Example 14.6. Let $X \equiv[0,1]$ and

$$
f \equiv\{(x, y) \in X \times X: 3 y=x \text { or } 3 y=x+2\} .
$$

Also, let

$$
C \equiv\{x \in X: x \text { has only 0's and 2's in its ternary expansion }\},
$$

i.e. the standard middle-third Cantor set on $[0,1]$. 
By the definitions it is easy to check that

$$
f^{\omega}=f^{\Omega}=\{(x, y) \in X \times X: x \in X, y \in C\} .
$$

We can see from here that $C$ is an attractor with dual repeller $\emptyset$. Also, $\emptyset$ is an attractor with dual repeller $X . \mathrm{C}(C)=C^{c}, \mathrm{C}(\emptyset)=\emptyset$ and we see that

$$
\mathrm{F}\left(f^{\Omega}\right)=\mathrm{R}(f)=\left(\bigcup_{A \in \mathfrak{A}(f)} \mathrm{C}(A)\right)^{c}=C .
$$

Also, $\mathcal{E}(f)=C \times C$ and we have one chain component: $C$.

\section{ThE QUADRATIC MAP}

The family of quadratic maps $f_{\mu}(x)=\mu x(1-x)$ on $[0,1]$ is a very well studied example for one-dimensional dynamics. We can find a thorough examination of the period-doubling route to chaos for this family in [5] or [10]. We will analyze now what happens to the Conley relation during this route.

Example 15.1. Let $X \equiv[0,1]$ and $f_{\mu} \equiv\{(x, y) \in X \times X: y=\mu x(1-x)\}$. It is not hard to show that if $1<\mu<3$, then $f_{\mu}$ has an attracting fixed point at $p_{\mu}=(\mu-1) / \mu$ and a repelling fixed point at 0 . In order to find $f_{\mu}^{\infty}, f_{\mu}^{\omega}$ and $f_{\mu}^{\Omega}$, we might invoke the help of a mathematical computer package. On Figure 1 we can see the first 15 iteration of $f_{\mu}$ for $\mu=2.8$.

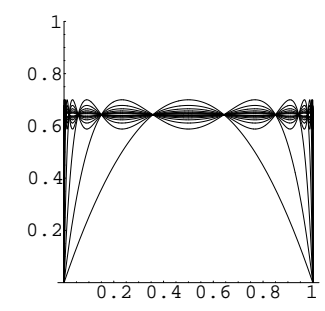

FiguRE 1. $\mu=2.8$

A very interesting question arises, which could be the topic of a lengthy investigation. What do we see exactly on this picture? Just by the definition of the limiting relations, we are tempted to say that the picture helps us to understand the structure of $f_{\mu}^{\infty}$ or $f_{\mu}^{\omega}$. However, after careful considerations of the processes used in the program, we can conclude that we get an insight to the properties of $f_{\mu}^{\Omega}$. Indeed, after analyzing the map we obtain Figure 2 for $f_{\mu}^{\Omega}$. It consists three line segments, a horizontal one for $0 \leq x \leq 1$ with value $(\mu-1) / \mu$ and two vertical ones, at $x=0$ and at $x=1$, with maximum value $\mu / 4$.

As we pass through $\mu=3$, the fixed point loses its stability and a stable period 2 orbit is born. The dynamics is the same for $3<\mu<1+\sqrt{6}$, let us pick the value $\mu=3.1$ to analyze the properties. On Figure 3 we can see the first 15 iteration of $f_{\mu}$ for $\mu=3.1$.

This changes $f_{\mu}^{\Omega}$ : it consists infinitely many line segments, two horizontal ones for $0 \leq x \leq 1$, with the values of the stable period 2 orbit, two vertical ones at $x=0$ and at $x=1$, with maximum value $\mu / 4$, and infinitely many vertical line segments at the preimages of the now unstable fixed point with values between the 


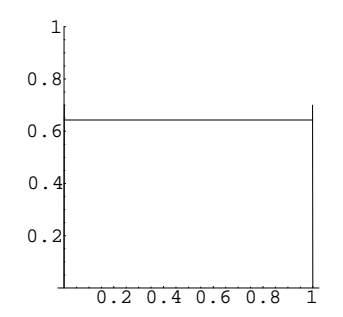

FigURE 2. $f_{\mu}^{\Omega}, \mu=2.8$

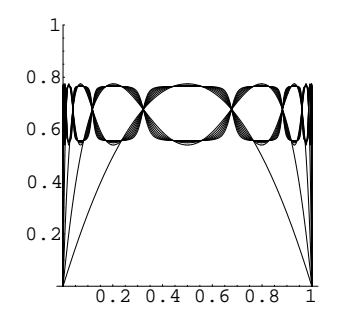

FiguRE 3. $\mu=3.1$

values of the stable period 2 orbit. Let us denote the nonzero unstable fixed point by $p_{0}$ and the stable period 2 orbit by $p_{1}$ and $p_{2}$.

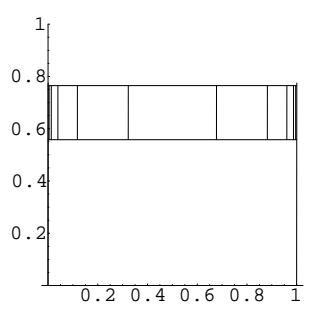

Figure 4. $f_{\mu}^{\Omega}, \mu=3.1$

We can graph $f_{\mu}^{\Omega}$ and $\left(f_{\mu}^{\Omega}\right)^{*}=\left(f_{\mu}^{*}\right)^{\Omega}$ together; this gives us an easy way to identify the attractors and corresponding dual repellers with the aid of Lemma 10.4 .

The attractor-repeller pairs are the following.

(1) $A_{1}=\emptyset, A_{1}^{*}=\left(f_{\mu}^{*}\right)^{\Omega}(X)=X$.

(2) $A_{2}=\left[p_{1}, p_{2}\right], A_{2}^{*}=\left(f_{\mu}^{*}\right)^{\Omega}\left(\left[0, p_{1}\right) \cup\left(p_{2}, 1\right]\right)=\{0,1\}$.

(3) $A_{3}=\left\{p_{1}, p_{2}\right\}, A_{3}^{*}=\left(f_{\mu}^{*}\right)^{\Omega}\left(X-\left\{p_{1}, p_{2}\right\}\right)=\left\{0,1, p_{0}\right\} \cup\left\{\right.$ preimages of $\left.p_{0}\right\}$.

(4) $A_{4}=[0, \mu / 4], A_{4}^{*}=\left(f_{\mu}^{*}\right)^{\Omega}((\mu / 4,1])=\emptyset$.

Also,

$$
\mathrm{F}\left(f^{\Omega}\right)=\mathrm{R}(f)=\left\{0, p_{0}, p_{1}, p_{2}\right\} .
$$

$\mathcal{E}(f)=\left\{(0,0),\left(p_{0}, p_{0}\right),\left(p_{i}, p_{j}\right): i, j=1,2\right\}$ and there are three chain components: $\{0\},\left\{p_{0}\right\}$ and $\left\{p_{1}, p_{2}\right\}$. 


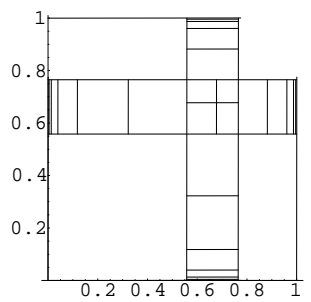

FiguRE 5. $f_{\mu}^{\Omega} \cup\left(f_{\mu}^{\Omega}\right)^{*}, \mu=3.1$

As $\mu$ is increasing, the period 2 orbit loses its stability and a period 4 stable orbit is born, etc. We know that the system goes through a period-doubling route to chaos. The period-doubling changes the picture of $f_{\mu}^{\Omega}$ in the above illustrated way. First the stable fixed point for $\mu=2.8$ corresponds to the horizontal line segment on the second picture. As $\mu$ passes through 3 , the horizontal line segment splits up into two horizontal line segments, which correspond to the period 2 stable orbit. The vertical line segments between are at the preimages of the now unstable fixed point. As $\mu$ passes through the next critical $\mu$ value, $1+\sqrt{6}$, the horizontal line segments split up again into two pairs of horizontal line segments, corresponding to the stable period 4 orbit, and between each pair new vertical line segments appear, at the preimages of the now unstable period 2 orbit. This process continues as $\mu$ passes through the consecutive critical values, where the stable period $2^{n}$ orbit loses its stability and a new $2^{n+1}$ period orbit is born, until $\mu$ reaches the value $\mu_{\infty} \approx 3.5699$.

\section{REFERENCES}

[1] E. Akin, The General Topology of Dynamical Systems, American Mathematical Society, 1993

[2] M. Barnsley, Fractals Everywhere, Academic Press, Inc., San Diego, 1988

[3] C. Conley, Isolated Invariant Sets and the Morse Index, CBMS Regional Conference Series 38, American Mathematical Society, 1978

[4] C. Conley, The gradient structure of a flow: I, Ergod. Th. and Dynamical Systems $\mathbf{8}^{*}$ (1988) $11-26$.

[5] R. Devaney, An Introduction to Chaotic Dynamical Systems, Benjamin/Cummings Publishing Company, Inc., 1986

[6] R. Langevin and F. Przytycki, Entropie de l'image inverse d'une application, Bull. Soc. Math. France 120 (1992) 237-250.

[7] R. Langevin and P. Walczak, Entropie d'une dynamique, C. R. Acad. Sci. Paris 312 (1991) $141-144$.

[8] R. McGehee, Attractors for Closed Relations on Compact Hausdorff Spaces, Indiana University Mathematics Journal, 41 (1992) 1165-1209.

[9] R. McGehee and E. Sander, A new proof of the stable manifold theorem, Z. Angew. Math. Phys. 47 (1996) 497-513.

[10] C. Robinson, Dynamical Systems, CRC Press, Inc., 1995

[11] E. Sander, Hyperbolic sets for noninvertible maps and relations, Discrete Contin. Dynam. Systems 5 (1999) 339-357.

[12] E. Sander, personal communication.

[13] T. Wiandt, Liapunov functions for closed relations, preprint

University of Minnesota, Minneapolis, MN

E-mail address: mcgehee@math.umn.edu 
Rochester Institute of Technology

E-mail address: tiwsma@rit.edu 